\title{
AUFSÄTZE
}

Klaus Schlichte

\section{Der Streit der Legitimitäten. Der Konflikt als Grund einer historischen Soziologie des Politischen}

Das so erfolgreiche Governance-Paradigma, so argumentiert der Beitrag, hat beträchtliche blinde Flecken. Aus einer Kritik seines negierten politischen Charakters lässt sich als positive Konsequenz die Forderung einer Rezentrierung des Begriffs des Politischen um den Konflikt als »Streit der Legitimitäten « ableiten. In der Unterscheidung zwischen Zuständen der Macht und Zuständen der Herrschaft, für die die Größe der Legitimität konstitutiv ist, lässt sich zudem eine verdeckte Konvergenz in der politischen Soziologie erkennen, die von Karl Marx über Max Weber und Norbert Elias bis zu Michel Foucault reicht. Aus dieser These von der Zentralität der Konflikte um legitime Geltung lässt sich zudem eine fachübergreifende Perspektive entwickeln, die für die Friedens- und Konfliktforschung, aber auch darüber hinaus neue Fragen aufwirft, zum Beispiel nach der Internationalisierung von Herrschaftszusammenhängen oder nach der Rolle des Gehorsams.

Schlagworte: Konflikt, Governance, Politikbegriff, Politische Soziologie, Gehorsam

\section{Der blinde Fleck der Governance}

Wenn man deutsche Einführungen und Lexika der Politikwissenschaft der jüngeren Zeit in die Hand nimmt, dann kann man den Eindruck gewinnen, es seien alle grundsätzlichen Fragen geklärt. Schlägt man darin etwa nach, wie Politik definiert wird, dann trifft man überraschend oft auf eine Definition, die wohl von vielen deutschen Politologen geteilt wird: Politik ist demnach »die Regelung der gemeinsamen Angelegenheiten eines Gemeinwesens durch allgemein verbindliche Entscheidungen « (Fuchs/Roller 2007: 205). Ein anderer Definitionsversuch adressiert zwar die Normativität, die jeder Definitionsversuch des Politischen unvermeidbar darstellt: Politik sei als $»$ Regelsetzung zu verstehen, an der all jene politisch beteiligt sind oder werden sollten, die der Herrschaft unterworfen sind « (Kreide/Niederberger 2011: 293). Auch diese Definition, die die Grenzen von Ordnungen immerhin 
noch thematisiert, teilt aber mit dem dominanten Diskurs der Politikwissenschaft die Zentrierung auf bewusste Entscheidungen und auf Institutionen.

Lange ließe sich die Liste ähnlicher Definitionen von Politik fortsetzen, in denen Ordnung immer schon vorausgesetzt wird und es nur noch um die - offenbar als friedliche Diskussion verstandene - Entscheidung über Regeln geht. Ganz offenbar sind diese Auffassungen von einer spezifischen Fassung der sozialen und politischen Realität geprägt, in der sie entstanden. Sie wirken wie der wissenschaftliche Ausdruck einer bürokratischen Institutionalisierung der Politik, der Herrschaft von Gremien und Ausschüssen. Der Boom der Governance-Literatur verstärkt diese Vermutung. Dieses prominente Paradigma versteht »Governance« entweder als »das Gesamt aller nebeneinander bestehenden Formen der kollektiven Regelung gesellschaftlicher Sachverhalte« (Mayntz 2004: 66) oder als Zusammenspiel von Institutionen, die der »Bereitstellung kollektiver Güter dienen« (Börzel 2010: 5). Trotz aller Evidenz kriegerischer Politik, Hegemoniebestrebungen und Asymmetrien, die international noch sichtbarer sind als in der Innenpolitik, wiederholt sich hier der Befund: Politik wird zur reinen Verfahrensfrage, in der es nur um das effiziente Arrangement von Institutionen geht.

Im Paradigma der »Governance « hat sich diese Auffassung inzwischen als Forschungsprogramm institutionalisiert: Der Begriff soll darauf aufmerksam machen, dass Steuern und Koordinieren immer häufiger »in horizontalen, netzwerkartigen Beziehungen zwischen öffentlichen und privaten Akteuren geschieht, wenngleich im Schatten des Staates« (Benz/Dose 2004: 22). »Governance« wird als Reformkonzept verstanden, das sich mit dem Leitbild des »aktivierenden Staates « verbinde, in dem nicht mehr der Staat, sondern die »Zivilgesellschaft« für gesellschaftliche Probleme zuständig sei. »Governance« bedeute also »Steuern und Koordinieren (oder auch Regieren) mit dem Ziel des Managements von Interdependenzen zwischen Akteuren, beruhend auf institutionalisierten Regelsystemen « (Benz/Dose 2004: 25). »Governance« diene dazu, »kollektive Sachverhalte zu regeln, Kollektivgüter bereitzustellen und politische Entscheidungen herbei zu führen « und dabei ließen sich »institutionalisierte Regelungsstrukturen« wie Hierarchie, Verhandlungssysteme und Wettbewerb unterscheiden (Risse 2007: 6). ${ }^{1}$

An diesem Paradigma kann viel kritisiert werden, und das ist auch schon geschehen. Sowohl der analytische Wert des Konzepts überhaupt (Offe 2009) wie auch

1 Das ist nicht neu, sondern die altbekannte Unterscheidung zwischen Wählen, Dekretieren und Verhandeln (vgl. Lehmbruch 1976). Im Unterschied zu diesen älteren Auffassungen der Politikwissenschaft kommen im Governance-Paradigma aber Diskurse, soziale Bewegungen, Opposition und Widerstand nicht mehr vor. Sie sind der liberalistischen Phantasie einer prästabilierten Harmonie zum Opfer gefallen. 
seine ideologische Funktion bei der Verschleierung von Interessenpolitik durch selbst ernannte Expertenkreise (Dobner 2010) sind thematisiert worden. Eigentümlich ist dem Ansatz aber auch, dass der politische Charakter des Modells selbst entweder gar nicht gesehen oder jedenfalls nicht weiter thematisiert wird. In den Augen mancher Autoren ist der Begriff »nicht mit einer bestimmten Theorie von Politik oder Gesellschaft verbunden« (Benz/Dose 2004: 27). Politik und »Governance« hingen insofern zusammen, »als das Politische alle auf Governance bezogenen Handlungen und Handlungszusammenhänge umfasst« (Risse 2007: 5). Der politische Charakter dieser Ordnungsvorstellung selbst wird gar nicht mehr diskutiert.

Dabei ließe sich durchaus fragen, inwiefern das Governance-Paradigma nicht bloß eine Verwässerung derjenigen alten Zentralbegriffe der politischen Theorie darstellt, die für die Legitimation moderner Demokratien einst tragend waren, wie die Gewaltenteilung, die Repräsentation oder das Gemeinwohl. Die Umstellung auf das »Management« von »kollektiven Gütern« scheint Verluste wesentlicher republikanischer Ideale mit sich gebracht zu haben (vgl. Gobin 2007).

Auch der Konflikt, die Auseinandersetzung und der Streit um die Berechtigungen und erst recht der Gebrauch von Zwang werden in dieser funktionalistischen Politikauffassung als dysfunktionale Reste überkommener hierarchischer Zeitalter und rückständiger Gegenden aufgefasst und höchstens therapeutisch thematisiert. Ganz offenbar dreht sich ein großer Teil der politikwissenschaftlichen Diskussion nur noch um die Frage institutioneller Arrangements, deren Modellierung weder historisch-kontextuelle Unterschiede kennt, noch Fragen der Legitimität jenseits von Input und Output behandeln will.

$\mathrm{Zu}$ vermuten, dass eine solche Auffassung von Politik selbst einem spezifischen Kontext geschuldet ist, scheint naheliegend, hält man dieses Verständnis etwa neben politische Realitäten anderer Zeiten in Deutschland oder die anderer Gegenden in der Gegenwart. Politik auf die »Herstellung kollektiv verbindlicher Entscheidungen « zu reduzieren muss auf jeden historisch Informierten unzulässig, wenn nicht absurd wirken. Weder der stark gewaltgeladene Prozess der Staatsbildung in Europa und besonders in Deutschland ist mit diesem Politikverständnis zu erfassen, noch sind es die Konflikte der Gegenwart. Die Politikwissenschaft, so scheint es, hat die Gewalt, den Zwang, den Konflikt in die Geschichte abgeschoben oder aber in jene nicht-westlichen Regionen, die nur hoffen können, ihre Rückständigkeit durch Beteiligung an Global Governance noch aufzuholen. Fachintern mag eine methodische und theoretische Engführung auf Varianten des Institutionalismus und rationalistische Politiktheorien, vor allem in Deutschland und den USA, zu dieser Entwicklung beigetragen haben. Kontexte, historische Verläufe, kurz: die Gesellschaftlichkeit 
und die Geschichtlichkeit politischer Gegenstände werden schlicht vergessen (vgl. Hagmann 2011).

Ein Verständnis des Politischen, das lediglich Prozesse der Entscheidungsfindung oder der Bereitstellung öffentlicher Güter als seinen Gegenstand erkennt, ist aber nicht nur ungeeignet, die politische Gegenwart oder die Geschichte zu begreifen. Das Governance-Paradigma ist, wie sich in den Konflikten der Europäischen Union und in jeder Parlamentsdebatte zeigt, eine idealistische Überformung, die weder die Wahrnehmung der Akteure abbildet, noch die öffentliche Diskussion um neue Beobachtungen bereichert. ${ }^{2}$

Stattdessen, so die These dieses Beitrags, bietet der Begriff des Konflikts einen viel geeigneteren Ausgangspunkt, eine interdisziplinär anknüpfungsfähige und theoretisch fruchtbare Perspektive auf politische Phänomene zu entwickeln. Vielleicht kann sich die Politikwissenschaft von der Konfliktforschung inspirieren lassen. Denn sowenig die Friedens- und Konfliktforschung im deutschen Sprachraum oder anderswo zu einer eigenständigen Disziplin geworden ist, ${ }^{3}$ so ist ihr Ausgangspunkt immer das Inkommensurable, das Nichtidentische und Nichtgleiche und sein Umschlag in widerstreitendes Verhalten geblieben. ${ }^{4}$ Der Gedanke, den Konflikt zum Ausgangspunkt von Theorien der Politik oder des Sozialen zu machen, hat zugleich lange historische Wurzeln. Dabei zeigen sich jedoch zwei grundsätzliche Richtungen, dieses Denken zu entwickeln. Entweder wird der Konflikt, das Ungeordnete, der Streit wie im kontraktualistischen Denken zum Anlass genommen, eine Ordnung zu begründen, also aus der Grundbeobachtung des Konflikts die Notwendigkeit von Herrschaft abzuleiten und die empirische Analyse so mit einer normativen Ambition zu verbinden. Oder aber die Grundbeobachtung des Konflikts führt dazu, ihn eben nicht still zu stellen, sondern als Modus von Politik überhaupt zu verstehen und seine Erscheinungsformen zu analysieren. In diesem Beitrag soll dieser zweite Impuls aufgegriffen werden, der vor allem soziologische Autoren angetrieben hat. Der Konflikt verdient es, von seinem schlechten Ruf befreit zu werden.

2 Nichts belegt die analytische Sprachlosigkeit der Governance-Politologie mehr als ihr Schweigen zur globalen Finanzkrise und zur Krise der Europäischen Union seit 2008. Das erstaunt besonders angesichts der enormen Ausweitung der europabezogenen Forschung, die sich das GovernanceParadigma so sehr zu eigen gemacht hat.

3 Zugleich hat die Konfliktforschung in Westeuropa und in Nordamerika eine lange Tradition, die jedoch vor allem in interdisziplinären Forschungsperspektiven bestand und besteht; vgl. hierzu Schlotter/Wisotzki (2011) und die Beiträge im Forum der Zeitschrift für Internationale Beziehungen, Heft $1 / 2012$.

4 Damit kann dieser Beitrag nicht beanspruchen, auch nur die wichtigsten theoretischen Bemühungen um den Konfliktbegriff aufzugreifen. Zu dieser enormen Vielfalt vgl. Bonacker (2002; 2009). 
Diese Perspektive des politischen und soziologischen Denkens lässt sich allgemein als historische Soziologie des Politischen charakterisieren, die in diesem Beitrag umrissen werden soll. Die erste zentrale These dieses Beitrags ist dabei, dass sich im »Streit der Legitimitäten« ein verdeckter Konvergenzpunkt bei einer Reihe von Autoren finden lässt, die gemeinhin als unvereinbar oder doch inkommensurabel einander gegenübergestellt werden. Auf der Grundlage des Vokabulars von Max Webers Herrschaftssoziologie werden Konvergenzen in den Werken von Karl Marx, Max Weber, Norbert Elias, Michel Foucault und Pierre Bourdieu im ersten Hauptteil dieses Beitrags diskutiert. Auch bei ihnen, so die These, finden sich analoge Überlegungen zu den hier an Weber entwickelten begrifflichen Unterscheidungen einer historischen Soziologie des Politischen. Die zentrale theoretische These ist dabei, dass sich der Begriff des Konflikts als Streit um legitime Geltungen auffassen lässt. Diese Auffassung ist an der Weberschen Unterscheidung zwischen Macht und Herrschaft als legitimer Macht orientiert. Der Streit um legitime Geltungen zeigt die Krise der Herrschaft an und gibt damit Auskunft über die Verteilungen, die von Ordnungen hergestellt werden. Im Konflikt zeigen sich aber auch Positionen, Argumente und Verhaltensformen, die über die Mechanismen der Macht und die Möglichkeiten ihrer Legitimierung, ihrer Verwandlung in Herrschaft Auskunft geben. Der Konflikt wird damit zum Ausgangspunkt der Analyse politischer Phänomene.

Der zweite Hauptteil dieses Beitrags wird die Reichweite und den spezifischen Gewinn der Perspektive einer historischen Soziologie des Politischen näher umreißen. Das Politische im Konflikt zu sehen und nicht so sehr im Zusammenwirken von Institutionen wie im Governance-Paradigma, hat vor allem für die politikwissenschaftliche Forschung innovative Konsequenzen. Vielleicht handelt es sich aber auch nur um die Erinnerung vergessener Bestände. Jedenfalls, das ist die zentrale These dieses zweiten Teils, erlaubt die historische Soziologie des Politischen die theoretisch anregende und analytisch fruchtbare Zusammenführung von sozialwissenschaftlichen Disziplinen, wie sie sich tatsächlich in der Friedens- und Konfliktforschung schon begegnen. Im Vordergrund steht dabei der Versuch, die Internationalisierung von Herrschaft auf neue Weise zu betrachten und dabei ausgehend vom Lokalen eine neue Analytik der Herrschaft zu entwickeln.

\section{Der Konflikt als Konvergenzpunkt einer historischen Soziologie des Politischen}

Die Konvergenz der politischen Soziologie besteht in der Zentralität der Unterscheidung von Macht und Herrschaft, wie sie in der Herrschaftssoziologie Max 
Webers (1985) am klarsten erkennbar ist. Herrschaft wird dabei als legitime Macht verstanden. Das Politische besteht im Konflikt, in den konfliktiven Übergängen zwischen Zuständen der Herrschaft und Zuständen der Macht. ${ }^{5}$ Mit dem Begriff der Herrschaft werden also solche Sonderformen von Macht bezeichnet, die legitime Geltung haben, häufig in einem Maße, dass sie gar nicht mehr erkennbar werden. Im Konflikt werden diese Geltungen bestritten, und im Konflikt entscheidet sich, ob sie weiterhin Geltung beanspruchen können oder ob neue Machtkonstellationen legitime Geltung erlangen.

Nun lassen sich weder Macht noch Herrschaft »an sich« empirisch einfach erfassen, weil sie beide entweder zu verbreitet sind (Macht) oder aber ihrer eingelebten Selbstverständlichkeit wegen unerkannt bleiben (Herrschaft). Erst im Konflikt, im Streit der Legitimitäten, in dem Herrschaftsansprüche behauptet, begründet, in Frage gestellt oder gänzlich bestritten werden und in dem Akteure machtvoll handeln, wird das Politische sichtbar. Im und am Konflikt lassen sich sowohl die Praktiken der Macht als auch die Strukturen der Herrschaft und die Diskurse der Legitimierung beobachten.

Für die historische Soziologie des Politischen scheint also zunächst der Begriff der Macht zentral zu sein. Doch jeder Begriff der Macht hat es in der empirischen Anwendung erstens mit dem Problem der Ubiquität der Macht und zweitens mit ihrer unendlichen Mannigfaltigkeit, ihrem »soziologisch amorphen « Charakter zu tun. Deshalb soll hier ein anderer Weg beschritten werden. Nicht der Begriff der Macht, sondern der des Konflikts bildet den Ausgangspunkt für eine Analytik des Politischen. Bevor diese Perspektive im Folgenden näher entfaltet wird, ist zur Verortung ein kurzer Blick auf etablierte Machtbegriffe hilfreich.

Machtphänomene lassen sich überall erkennen, die Macht ist anscheinend ubiquitär. Dadurch wird unklar, welche sozialen Beziehungen und welche Phänomene analytisch isolierbar sind. Wenn alles von der Macht durchzogen ist, dann gibt es keinen aus der Theorie oder aus der Erfahrung begründet privilegierten analytischen Zugang zu ihrer Analyse. Alles und jedes kann in den Blick genommen werden.

Neben die Ubiquität der Macht tritt als zweite Schwierigkeit ihr amorpher Charakter. Zwar mangelt es nicht an Typologien von Machtbegriffen, mit denen die politische und soziologische Theorie versucht hat, Machtphänomene zu ordnen. So

5 Diese Bezeichnung knüpft an die Terminologie Jacques Rancières an, der Politik auf die Emergenz des Konflikts reduziert wissen will, während für ihn alle verwaltende und regierende Tätigkeit stattdessen »Polizei« ist (Rancière 2002: 39-45). 
wurden akteurszentrierte und systemorientierte Machtbegriffe unterschieden. ${ }^{6}$ Die Webersche Definition des Machtbegriffs steht demnach im Gegensatz zur Fassung der Macht als symbolisch generalisiertem Kommunikationsmedium in der Theorie sozialer Systeme.

Eine andere Unterscheidung wurde mit power over im Gegensatz zu power to gekennzeichnet (Pitkin 1972). Im Sinne des letzteren Verständnisses haben insbesondere Hannah Arendt und Talcott Parsons Macht als Gegenbegriff zur Gewalt verstanden (vgl. Clegg/Haugaard 2009: 3). Gerhard Göhler hat zum Verständnis von Machtkonzeptionen viel mit seiner Unterscheidung von transitiver versus intransitiver Macht beigetragen, die als komplementäre Seiten seines Machtbegriffs verstanden werden könnten. Macht wird dabei als Medium sozialer Beziehungen verstanden, das Handlungsräume strukturiert (Göhler 2009: 36; 2011: 237). Damit ist aber noch kein analytischer Ausgangspunkt für die empirische Forschung gewonnen.

Es ist wohl nicht zu erwarten, dass eine Analytik der Macht jemals zu so etwas wie einer erschöpfenden Bestimmung des Machtbegriffs oder vollständigen Erfassung empirischer Machtphänomene führen würde. Die Macht wird ihr Enigmatisches behalten, wohl in höherem Maße als die Phänomene der Herrschaft oder der Legitimität. Aber das spricht nur dafür, sie gerade deshalb als zentralen Gegenstand der wissenschaftlichen Beschäftigung mit Politik zu bewahren, auch wenn sie nicht der analytische Anfangspunkt sein kann.

Eine sowohl theoretische wie empirisch-pragmatische Alternative zur Zentrierung der politischen Soziologie um den Begriff der Macht bietet der Begriff des Konflikts. Konflikte sind empirisch beobachtbare Widerspruchskommunikationen, die episodenhaft bleiben oder sich zu Strukturen verfestigen und gewaltsam eskalieren können (vgl. Messmer 2003). In Sprache, Gestik und Mimik, in beobachtbarem Verhalten wird aber zugleich Inhaltliches transportiert. Konfliktparteien erheben Ansprüche, sie bestreiten und behaupten Gründe, sie schreiben Schuld zu, verneinen und begründen Berechtigungen. Noch der Gebrauch physischer Gewalt ist immer zugleich Kommunikation im Konflikt. ${ }^{7}$

6 In den posthum zusammengestellten Arbeiten von Niklas Luhmann finden sich zu dem Ausdruck »legitime Macht« Ausführungen, die diesen ganz in die Nähe des Weberschen Herrschaftsbegriffs rücken (vgl. Luhmann 2010: Kap. 18). Das unterscheidet sich stark von der weniger kompatiblen Fassung der Macht als »symbolisch generalisiertem Kommunikationsmedium« in früheren Werken. Gibt es auch hier eine Konvergenz?

7 Diese Einsichten verdanken sich den anregenden Diskussionen des »Netzwerks Konstruktivistische Konfliktforschung« (vgl. http://netzwerk-konfliktforschung.de/). 
Konflikte zeigen damit die Krise von Legitimitäten an. Wo Herrschaftsformen selbstverständlich geworden sind, wird kein Widerspruch zu ihnen kommuniziert. Im Konflikt werden Legitimitäten aber auch erzeugt: Der Widerspruch nötigt selbst zur Begründung und im Streit entstehen so neue Muster der Legitimierung, deren Erfolg aber unsicher ist. Entlang der Weberschen Terminologie lässt sich diese Auffassung von der analytischen Zentralität des Konfliktbegriffs am ehesten explizieren, denn es sind eben die Übergänge zwischen Zuständen der Herrschaft und Zuständen der - nicht-legitimen - Macht, die der Konflikt bezeichnet und die den Kern des Politischen bilden.

Max Weber hat den Begriff der Herrschaft bekanntlich als legitimierte Macht aufgefasst,${ }^{8}$ für die besonders die Beziehung zwischen dem »Herrn« und dem Apparat sowie die Beziehung beider zu den Beherrschten strukturbildend ist (Weber 1985: 549). Herrschaftsbeziehungen sind solche, in denen die Asymmetrie akzeptiert und eingeübt ist. Muster der Interaktion laufen ab, ohne Fragen nach Begründungen aufzuwerfen. Regeln sind habituell verankert und erlauben damit das mehr oder weniger reibungslose Funktionieren von Institutionen und Organisationen.

Herrschaft, so sagt Weber selbst, ist eine Sonderform von Macht (Weber 1985: 541). Was Formen der Herrschaft von einfacher Macht unterscheidet, ist ihre Legitimität, die Institutionalisierung ermöglicht. In den bekannten Definitionen der Begriffe von Macht und Herrschaft tritt dieser Unterschied deutlich hervor: Macht wird dabei verstanden als »jede Chance, in einer sozialen Beziehung den eigenen Willen auch gegen Widerstreben durchzusetzen«, während Herrschaft von Weber definiert wird als »die Chance, für einen Befehl bestimmten Inhalts bei angebbaren Personen Gehorsam zu finden«. Der »angebbare Personenkreis« und die Worte »Befehl« und »Gehorsam « weisen bereits auf den institutionalisierten Charakter der Beziehung hin. Das Zeigen der Machtmittel, die Drohung, ist nur noch im Grenzfall nötig und indiziert eigentlich schon einen Übergang zum Konflikt. Im Alltagsfall, so Weber weiter, ist Herrschaft aber einfach Verwaltung. Ein Verwaltungsstab erledigt routiniert die standardisierten Aufgaben, die Hierarchien sind eingelebt und weder Herrscher noch Beherrschte stellen die Geltungsgründe der »legitimen Ordnung « in Frage. ${ }^{9}$

8 Diese Interpretation findet sich u.a. bei Philippe Reynaud (1996: 159-160). Am detailliertesten hat Hartmann Tyrell (1980) diese Interpretation entwickelt.

$9 \mathrm{Im} \S 5$ der »Soziologischen Grundbegriffe« zur »legitimen Ordnung« stellt Weber den Begriff der Legitimität noch einmal in einen breiteren sozialen Zusammenhang als in der Herrschaftssoziologie. Aber auch hier wird Legitimität als Glauben an die »Vorbildlichkeit oder Verbindlichkeit« der gegebenen Ordnung von rein zweckrationalen Motiven oder der traditionalen Sitte unterschieden (Weber 1985: 16). 
Zwar beruhen Herrschaftsbeziehungen nicht allein auf dem Glauben an die Geltung einer Ordnung. Weber hat zwei weitere zentrale Elemente stabiler Herrschaftsbeziehungen genannt, nämlich zum einen die »Übereinstimmung mit der Sitte « und zum anderen die »Erfüllung des materiellen Interesses mindestens des Verwaltungsstabes «. Beide allein aber, so Weber, können nur labile Herrschaftsverhältnisse begründen (Weber 1985: 122). Stabilere Formen politischer Herrschaft haben so zwar zur Voraussetzung, dass die politische Ordnung nicht in offenem Widerspruch zum eingelebten moralischen Kanon steht - ein chronisches Problem vieler Fremdherrschaften - und dass das Versorgungsinteresse der Mitglieder des Apparates einigermaßen befriedigt wird. Aus der Sicht der Weberschen Herrschaftssoziologie handelt es sich hierbei allerdings eher um Gemeinplätze. Die soziologisch interessante Frage ist die nach der Legitimität als dritte Voraussetzung stabiler politischer Herrschaft. Das Bemerkenswerte an der Legitimität sind beide ihrer Seiten: Ihre vermeintliche Normalität ist nach wie vor genauso wenig bestimmt wie die Mechanismen ihrer Krise.

Die spezifische Funktion der Friedens- und Konfliktforschung besteht darin, diesen Streit der Legitimitäten, das Feld des Konflikts näher zu bestimmen. Denn für politischen Wandel, für die Erosion von Institutionen der Herrschaft wie für deren Entstehung, ist die Dynamik des »Geltungsglaubens«, die Frage der Legitimität, zentral. Dies ist jedoch keine rein theoretische Deduktion, sondern zugleich eine ubiquitäre Beobachtung: Streit um Begründungen und rationale Argumentationen werden in den Sozialwissenschaften als konfliktiver Prozess der Generierung von Legitimität anerkannt. »Datensetzende Macht« oder die »Aktionsmacht« physischen Zwangs (Popitz 1992) werden hingegen als das Gegenteil von Legitimität verstanden, obwohl in der Genealogie von Herrschaftsbeziehungen beides, Legitimität und Disziplinierung, schwer zu trennen ist.

\section{Von Marx zu Foucault - die Protagonisten einer Konvergenz?}

Dass Herrschaft legitime Macht ist und dass sich ihre Ansprüche in Konflikten erst durchsetzen müssen und dass in diesem Prozess Legitimierungen und Disziplinierungen stattfinden, das ist jedoch nicht nur Max Webers Ansicht. Auch an den theoretischen Arbeiten und den empirischen Analysen anderer »klassischer« Autoren der politischen Soziologie lässt sich eine damit kompatible, wenn nicht in Grundformen identische Orientierung erkennen. Auch andere Autoren konvergieren mit Weber darin, dass nicht einfache, krude Macht, die auf gleichzeitige Anwesenheit angewiesen ist, hinter der Geltung einer Ordnung stehen kann. Die Verstetigung dieser Macht durch ihre Institutionalisierung ist vielmehr teils ein Werk der Diszi- 
plinierung und teils ein Werk der Legitimierung. Durch beide Vorgänge wird aus Macht Herrschaft.

Das soll im Folgenden an vier Autoren gezeigt werden. Zugleich soll dieser Abschnitt dazu dienen, aus den Konzeptualisierungen des Zusammenhangs von Macht und Herrschaft der diskutierten Autoren einige zentrale Elemente einer historischen Soziologie des Politischen zusammenzutragen, die im nachfolgenden Abschnitt im Hinblick auf neue Forschungsperspektiven der Friedens- und Konfliktforschung diskutiert werden. Die Unterschiedlichkeit der hier nur kursorisch behandelten Autoren macht zugleich deutlich, in welcher Varianz eine historische Soziologie des Politischen möglich ist.

Der Verweis auf »Klassiker« soll also nicht der »charismatischen Rezeption« Vorschub leisten (vgl. McFalls et al. 2007: 14), sondern lediglich zeigen, dass diese besonders einflussreichen theoretischen Bezugnahmen nicht in dem Verhältnis vollständiger Unvereinbarkeit zueinander stehen, das der Schulenstreit Theorien oft zuschreibt, ohne dabei die unter Umständen verzerrende Rolle der je eigenen Werkinterpretationen einzurechnen (vgl. Colliot-Thélène 2001: 3).

Im Mittelpunkt der Diskussion um die Formen und den Wandel politischer Herrschaft steht bis heute der Staat. Grund dafür ist nicht nur seine Verallgemeinerung zur global dominanten politischen Organisationsform und seine Persistenz in Zeiten der Globalisierung. Der wichtigste Grund liegt darin, dass auch die wissenschaftlich-theoretische Sprache tief von der staatlichen Organisation und der sie begleitenden Semantik geprägt ist.

In der Analyse der Legitimität staatlicher Herrschaft findet sich so auch der erste und wichtigste Konvergenzpunkt der hier nur kursorisch behandelten Autoren. Sie alle thematisieren den Streit der Legitimitäten vor allem am Staat. Der »Geltungsglaube « wird dabei von allen im Anschluss diskutierten Autoren als Resultat sozialer und politischer Auseinandersetzungen gesehen und damit historisiert. Der Staat ist keine überzeitliche und durch sein Recht selbst legitimierte quasi-theologische Größe, sondern ein Herrschaftsgebilde, das in langen Machtkonflikten geformt wurde und dessen Trägerschichten selbst eine Legitimationspolitik betreiben. Die Geschichte staatlicher Herrschaft ist zugleich die Geschichte des Wandels der von ihm beherrschten Subjekte und Diskurse.

So umfasst Webers Staatsbegriff entgegen landläufiger Darstellungen nicht bloß das Gewaltmonopol, sondern er ist genauer definiert als ein politischer Verband, der ein Monopol auf den Gebrauch der Zwangsmittel beansprucht und dafür Legitimität erwirbt (Breuer 1994: 24-26). ${ }^{10}$ Zugleich ist aber auch die Rekonstruktion

10 Wie genau Staaten und andere politische Organisationen Legitimität erlangen, ist eine für die Gegenwart erst in Anfängen bearbeitete Forschungsfrage. Zum westlichen Staat und den von ihm 
dieser spezifischen Form politischer Herrschaft ein Hauptthema von Webers Herrschaftssoziologie (vgl. Breuer 2007). Die Herausbildung des Beamtentums als einer spezifischen Trägerschicht staatlicher Herrschaft wie die disziplinierende Funktion staatlicher Gewaltapparate und Erziehungseinrichtungen ergänzen die Legitimationspolitik staatlicher Instanzen. Die Rationalisierung staatlicher Herrschaft, wie sie im Recht und in der Verwaltung erkennbar wird, ist Webers Analyse zufolge aber vor allem das Ergebnis äußerer Einflussnahme und von der Geschichte des modernen Kapitalismus nicht zu trennen (Weber 1991: 293). Der Monopolisierungsanspruch der Ordnungsfunktionen und der Drang zur Rechtsförmigkeit sind zugleich funktionale Notwendigkeiten der Massengesellschaft und erhoffte Legitimitätsquellen (Anter 2001: 124). Eine solche Einbettung des Formwandels politischer Herrschaft in größere soziale Prozesse und soziale Interessenlagen durchzieht die politische Soziologie durchweg (vgl. Schlichte 2005: Kap. 2.1).

Mit der hier behaupteten Konvergenzthese ist gemeint, dass eine Vielzahl von mittlerweile klassischen Autoren der politischen Soziologie in der Analyse politischer Phänomene eine ähnliche Perspektive einnimmt. Auch bei Karl Marx, Norbert Elias, Michel Foucault und Pierre Bourdieu werden Phänomene der Macht von Herrschaftszuständen unterschieden, auch bei ihnen liefern die Konflikte die analytischen Zugriffe auf die Anatomie politischer Herrschaft. ${ }^{11}$ In ihren Beiträgen lässt sich also eine Konvergenz mit den Grundverständnissen der Weberschen Herrschaftssoziologie erkennen.

\subsection{Karl Marx und die Ideologie des Staates}

Die etablierte Entgegensetzung von Marx und Weber hat lange verdeckt, dass die Kritik eigenlegitimatorischer Figuren staatlicher Herrschaft durch Marx auf eine mögliche Konvergenz seiner Auffassungen politischer Herrschaft zur Weberschen Perspektive verweist. Statt den Staat als wirkende Idee zu setzen, so Marx, sei seine Genese aus dem Handeln der wirklichen Menschen zu untersuchen, weil der Staat sonst als »illusorische Gemeinschaftlichkeit« erscheint (Marx, MEW 3: 33; vgl. Reichelt 2008). Ganz wie im Programm der Weberschen Herrschaftssoziologie for-

getragenen internationalen Organisationen vgl. Biegoń et al. (2010), zu regionalspezifischen Ansätzen vgl. z.B. Schatzberg (1980), zu autoritären Staaten Hibou (2011). Rodney Barker (1990) vertritt die originelle These, dass ein Großteil der Legitimationspolitik nicht der Generierung von Legitimität bei den Beherrschten dient, sondern auf die Trägerschichten und politischen Klassen selbst zielt, also der Eigenmotivierung dient.

11 Diese Konvergenzen lassen sich in den Werken der späteren politischen Soziologie natürlich ebenso bemerken, etwa bei Charles Tilly, Theda Skocpol oder Anthony Giddens (vgl. Mann 1984; Bhambra 2010). 
dert Marx, nicht abstrakte Ideen, sondern die konkreten sozialen Auseinandersetzungen zu analysieren, die über die Strukturen der politischen Herrschaft und auch ihre legitimierenden Diskurse entscheiden (vgl. Colliot-Thélène 2001: 17).

Auch im Staat, also im Feld der politischen Herrschaft, finden Kämpfe statt (Marx, MEW 3: 33). Der Staat wird also keineswegs als bloß funktionales Erfordernis der bürgerlichen Klassenherrschaft theoretisch deduziert, sondern die Analyse politischer Herrschaft ist auch bei Marx schon soziologisch, indem nach Gruppen, ihren Interessen, ihren Besonderheiten und ihren Interaktionen gefragt wird.

Der Staat als »legitime Ordnung« erscheint bei Marx, wie später bei Gramsci, auch als eine »geistige Macht « (Marx, MEW 3: 46). Jede politische Herrschaft gibt Marx zufolge ihre Interessen als allgemeine aus, und diese Allgemeinheit ist umso größer, je mehr Interessen dabei berücksichtigt werden können (Marx, MEW 3: 47, Fn. 17).

Auch andere wesentliche Argumente der kritischen Analyse politischer Herrschaft, wie sie bei Max Weber und Pierre Bourdieu entfaltet werden, liegen bei Marx schon vor: Er kritisiert die Bürokratie als »Illusion des Staates" (Marx 1953: 60) und das Beamtentum, ihre Trägerschicht als »Staatsjesuiten und Staatstheologen «. Das Recht ist für Marx noch ebenso Ideologie wie die sonstigen Diskurse des Staates. ${ }^{12}$ Auch hier ist Marx nahe an der Kritik der Verselbständigung bürokratischer Herrschaft durch Weber (Weber 1985: 572): »Die Staatszwecke verwandeln sich in Bürozwecke oder die Bürozwecke in Staatszwecke« (Marx 1953: 60). Die Autorität ist das Prinzip ihres Wissens und die Vergötterung der Autorität ist die Gesinnung der Bürokratie (Marx 1953: 61). Wie Weber, so kennt also auch Marx die Arbeit der Legitimierung staatlicher Herrschaft im modernen Staat und die Gruppen, die diese vornehmlich betreiben: Politiker, Juristen und Bürokraten, deren geistige Welt sich aus ihrer praktischen Lebensstellung, ihrem Geschäft und der Arbeitsteilung erklären (Marx, MEW 3: 50).

Der Wandel politischer Herrschaft wird auch bei Marx über den Verweis auf konfliktive historische Prozesse erklärt, und dieser Wandel, ob inkremental, in Reformen oder Revolutionen, ist nicht bloß eine Veränderung von Kräfteverhältnissen, sondern steht in Zusammenhang mit der Veränderung ganzer Lebensverhältnisse. Deshalb muss die Analyse politischer Herrschaft aus dieser Perspektive mehr tun, als nur Institutionen in den Blick zu nehmen. Sie muss die Formen und Praktiken

12 Zur Entwicklung späterer Rechtsauffassungen auf der Grundlage von Marx’ Werk, die den »Kompromisscharakter« des Rechtsstaats für lebensfähig halten, etwa bei Hermann Heller, Franz Neumann oder Otto Kirchheimer, vgl. Yildiz (2008). 
der Herrschaft historisieren, die »Trägerschichten« (Weber) bestimmen und die Diskurse der Herrschaft kritisch rekonstruieren.

Zwei epochale soziale Prozesse subsumiert Marx dabei im Begriff der bürgerlichen Gesellschaft, und beide Prozesse haben ihre Entsprechungen im Programm der Weberschen Soziologie. Zum einen historisiert auch Marx das bürgerliche Subjekt in einer Weise, die dem »rationalen Wirtschaftsethos« Webers (Weber 1991: 302) sehr nahe kommt. Bei Marx heißt dies das bürgerliche Subjekt, das von individuellen Interessen regiert wird. Seine charakteristische Motivationsstruktur, die »Privatzwecke«, werden jedoch erst mit der bürgerlichen Gesellschaft, also ab dem 18. Jahrhundert, zur historisch bestimmenden Kraft (Marx, MEW 42: 20).

Der zweite große Prozess, den Marx im Begriff der bürgerlichen Gesellschaft eher versteckt als explizit macht, ist die Einbettung politischer Herrschaft in den Weltmarkt, der zur Konstitutionsbedingung politischer Herrschaft in der kapitalistischen Moderne wird (Marx, MEW 42: 188). ${ }^{13}$ Bei Max Weber erscheint diese Internationalität hingegen in der Hochzeit des Imperialismus nur als Konkurrenz der Machtstaaten (vgl. Weber 1988: 158).

Diese notwendig kursorischen Parallelisierungen lassen ein erstes Resümee des Programms einer historischen Soziologie des Politischen zu: Marx und Weber teilen das Postulat, die Befunde über Strukturen, Werte und Institutionen politischer Herrschaft auf die Analyse »sozialer Trägerschichten « zurückzubinden. Es reicht demnach nicht, in theoretischer Sprache auf höherer Abstraktionsebene Strukturen und Verhältnisse zu benennen, sondern eine wirklich sozialwissenschaftliche Analyse muss auch die Akteure und Konstellationen identifizieren können, denen sich die Ausbildung und der Gehalt von Strukturen verdankt. In dieser Hinsicht trifft sich das Webersche Programm mit den Anforderungen, die auch Marx an die Rekonstruktion von historischen Verläufen und kritischer Auseinandersetzung mit der Gegenwart stellt, nämlich als »Reproduktion des Konkreten im Wege des Denkens« (Marx, MEW 42: 35). Diese Analyse muss zugleich historisch verfahren, denn politische Herrschaft folgt nicht transhistorisch universellen Gesetzmäßigkeiten, sondern die Konstellationen sind historisch vielfältig, die Formen der Legitimität ebenso. Die Zusammenhänge können deshalb nicht theoretisch deduziert werden, sondern müssen in Vermittlung zum historischen, empirischen Primärmaterial rekonstruiert werden. Diese Historizität erstreckt sich auch auf die Subjekte, die an poli-

13 Diese grundlegende These eint bis heute eine Vielzahl von sonst sehr unterschiedlichen vom Marxschen Werk inspirierten Autoren (vgl. Krippendorff 1975; Senghaas 1982; Bayart 2000). Dass die Formen der Einbettung in den Weltmarkt auch als Formen der Herrschaft aufgefasst werden können, wird außer von der Dependenztheorie auch von den postcolonial studies vertreten (vgl. Iggers et al. 2008). 
tischer Herrschaft beteiligt sind und ihr zugleich unterliegen: Handlungsrationalitäten liegen nicht transhistorisch fest, sondern verändern sich ebenso in Wechselwirkung mit historischen Prozessen und den Effekten politischer Herrschaft, weil sich mit ihrer Form auch die Subjektivitäten verändern. Dieser letzte Punkt ist das Zentralthema der politischen Soziologien von Norbert Elias und Michel Foucault.

\subsection{Norbert Elias - Macht und Herrschaft in Figurationen}

Im Werk von Norbert Elias finden sich zwar eine Reihe von kritischen Bemerkungen zu Webers methodologischem Individualismus. Sein Verständnis von Macht als Eigenschaft von sozialen Beziehungen aber zeigt eine große Nähe zum Weberschen Verständnis. Auch Elias hebt hervor, dass die Quellen der Macht polymorph sind. Es gibt für ihn keine feststehende Grenze dafür, was in Beziehungen zum Mittel der Macht wird (Elias 1970: 97). Wie Weber denkt auch Elias Macht nicht substanzhaft, sondern relational. Macht ist bei Weber eine Chance, also eine Wahrscheinlichkeit, dass sich in einer Beziehung etwas ereignet. Das deckt sich mit dem Eliasschen Verständnis von Macht als einer »Struktureigentümlichkeit menschlicher Beziehungen « (Elias 1970: 77). Doch auch bei Elias findet man Formulierungen, in denen die Macht als Chance bezeichnet wird (Elias 1987: 80).

Der Machtbegriff Webers hat eine strukturelle Voraussetzung, die Weber mit dem Begriff der »sozialen Beziehung« definiert hat: das gegenseitig aufeinander eingestellte soziale Handeln der Interaktionspartner (vgl. Kaven 2006: 42-63). Die Ausübung von Macht ist also auch bei Weber nicht voraussetzungslos, die Konstitution von Gesellschaftlichkeit ist ihr immer schon vorgängig. Elias betont aber stärker noch als Weber den Beziehungscharakter der Macht. Er fügt dem ein Spezifikum hinzu, nämlich dass diese Beziehungen immer als Machtbalancen zu sehen seien. Immer stehe der Macht des einen die Gegenmacht des anderen gegenüber. Aus diesem Grundverständnis der Macht entwickelt Elias seinen Begriff der Figuration, die er als Netz interdependenter Beziehungen mit ihren Machtbalance charakterisiert. Dem absolutistischen Königshof ist dazu die prominenteste Studie von Elias gewidmet.

Eigentümlicherweise spielt der Begriff der Herrschaft in Elias' Arbeiten keine Rolle. In den Sachregistern seiner Werke taucht das Wort nicht auf. Ein Grund dafür könnte Elias' konstantes Insistieren auf dem Prozesscharakter von Machtphänomenen sein. Denn für ihn ist die Macht nichts Konstantes. Alle Machtbalancen, aus denen Figurationen bestehen, sind ständig in Bewegung, auch wenn diese Bewegungen oft kaum wahrnehmbar sind. Schließlich stehen die Beziehungen untereinander in einem Verhältnis: Die Geflechte von Interdependenzen zwischen Men- 
schen und Gruppen bedeuten ein ständiges Spiel der Veränderung. Allenfalls diese verschränkten Interdependenzen lassen sich als Elias' Variante des Herrschaftsbegriffs interpretieren.

Gleichwohl sind Phänomene legitimierter Macht Elias nicht unbekannt. Sie spielen in seinem Werk sogar eine prominente Rolle. In seiner Arbeit über die englische Kleinstadt Winston-Parva, in der es um die Konstruktion der Beziehung von Etablierten und Außenseitern geht, kommen Figuren der Legitimität vor, der symbolischen Umkleidung von Beziehungen, bis die behaupteten Unterschiede im Denken der Beteiligten verdinglicht sind und sie als symbolische Ordnung beherrschen (Elias/Scotson 1990: 8-12). In seiner Analyse der politischen Geschichte Deutschlands kommt der Verherrlichung der Kriegerehre als legitimierender Figur eine zentrale Rolle zu (Elias 1990: 69). Und natürlich ist das ganze Thema seines Frühwerks eine Geschichte der Legitimierung von Macht: Die Monopolisierung der Gewalt und die Absicherung dieser Chancen durch die symbolischen Ordnungen der königlichen Höfe sind die zentralen Vorgänge der Entstehung des neuzeitlichen Staates und der formalen Rationalisierung von Herrschaft im modernen Staat (vgl. Bogner 1989; Heinich 1997).

Für den Übergang von Macht zu Herrschaft, der also im Werk von Elias einen prominenten Platz einnimmt, spielt neben der symbolischen Ordnung aber noch ein zweiter Weberscher Begriff eine große Rolle, die Disziplinierung. Bei Elias bleibt letztlich unklar, ob die Legitimierung einer Ordnung durch semantische Arbeit erzielt wird oder ob nicht die Disziplinierung, bei Elias gefasst als die »Verinnerlichung von Fremdzwängen« dabei eine größere Rolle spielt. Den Zwangscharakter der Strukturierung von Gesellschaft und der Subjektivierung des einzelnen hat er jedenfalls nicht übersehen. Mit dem Stichwort der Disziplinierung ist zugleich eine Korrespondenz in den politischen Soziologien von Norbert Elias und Michel Foucault benannt.

\subsection{Michel Foucault - Macht und Subjekt}

Dass es Parallelen zwischen den Machtverständnissen von Weber und Foucault gibt, ist bereits mehrfach hervorgehoben worden (vgl. McFalls 2008; Colliot-Thélène 2001). ${ }^{14}$ Dennoch fällt es aus zwei Gründen schwer, Foucaults Machttheorie in die hier behauptete Konvergenz - nämlich in der Zentralität des Konflikts um Legitimitäten - einzuordnen. Erstens kann es laut Foucault keine transhistorische Theorie der Macht geben, die Macht ist ihm zufolge immer ein »Beziehungsknäuel« (Fou-

14 Solche Konvergenzen sieht auch Thomas Lemke (2001), der hingegen auch Unvereinbarkeiten zwischen den Perspektiven von Weber, Foucault und Elias betont. 
cault 1987: 216). Sie ist das Wirken von Technologien und die Resultate dieses Wirkens, sie ist eine Matrix (Foucault 1987: 217) und damit erscheint das Verständnis von Macht bei Foucault noch einmal eine Stufe abstrakter als bei Elias. Während Elias die Macht als Merkmal von Beziehungen in Figurationen bestimmt, ist Foucaults Machtbegriff unendlich schillernd. Nicht einzelne Beziehungen, sondern immer die Ordnung als Ganze, das Ensemble von Praktiken und Diskursen, ist »die Macht«. Sie ist eine Matrix von jeweils konkreten, aber historisch wandelbaren Konstellationen.

Entsprechend finden sich bei Foucault unendlich viele Bestimmungen des Begriffs und fast zu jeder Bestimmung an anderer Stelle der entsprechende Widerspruch dagegen. Die Macht ist auch bei ihm ubiquitär und polymorph: Mal ist sie Struktur, dann Aktion, mal Effekt und mal Wissen, mal Diskurs. Schließlich ist sie auch das Subjekt selbst, und am überzeugendsten wird Foucault da, wo er feststellt, dass seine Machttheorie eigentlich eine Theorie des Subjekts ist. Die Kategorie des assujettissement - die soziale Produktion des sich autonom dünkenden Subjekts, das zugleich Unterworfener ist - scheint der Schlüssel zum Verständnis der politischen Soziologie im Werk Foucaults zu sein. Das Subjekt ist selbst ein Effekt der Macht. ${ }^{15}$ Durch diese Auflösung des Subjekts wird Foucaults Machttheorie auf den ersten Blick inkompatibel mit dem Weberschen Verständnis, das weniger die Subjekte als die »Chance« innerhalb von Beziehungen akzentuiert.

Der zweite Grund, warum es schwer zu fallen scheint, Foucault in die hier behauptete Konvergenz einzuordnen, liegt darin, dass der Begriff der Herrschaft bei ihm offenbar keine zentrale Rolle spielt. Die Legitimität ist schon gleich ein Begriff, der bei Foucault von der Kategorie der Disziplin vollständig verdrängt wirkt. Gerade in seinen materialreichen, empirienächsten Arbeiten wirkt das Zwanghafte, die Disziplin, übermächtig. Die Anstalten, ihre Techniken und die sie konstituierenden Diskurse und Praktiken scheinen so etwas wie Legitimität gar nicht zu kennen und nicht zu brauchen.

Aber auch bei Foucault ist nicht alles feste Struktur. Es gibt auch den Konflikt, die Herausforderung der Ordnung, oder, um bei seiner Diktion zu bleiben: der Macht. In Konflikten wird die Macht beweglich, sie wird herausgefordert, um dann freilich nur wieder in neuen Machtordnungen zu ändern. Diese neuen Sedimentierungen sind durchaus produktiv (Foucault 2005: 238). In Konflikten findet also etwas zum Weberschen Verständnis Analoges statt: Die legitime Ordnung wird

15 Es ist diese Historisierung des Subjekts, die vor allem in der Politikwissenschaft bisher am wenigsten zur Kenntnis genommen wurde, vielleicht weil sie zu viele Prämissen der Modellierung von Politik in Frage stellen würde (vgl. Brass 2000). Zum Zusammenhang von Subjektbildung und globaler Vergesellschaftung vgl. Bayart (2004). 
herausgefordert, aber die Konflikte enden in der Regel in einer neuen legitimen Ordnung. Wiederum wird aus Macht eine Herrschaft.

Im Vokabular Foucaults gibt es schließlich nicht nur die pouvoir, sondern auch die états de domination, also Herrschaftszustände. In seiner programmatischen Auseinandersetzung mit dem Konzept der Genealogie Nietzsches finden sich dazu aufschlussreiche Passagen (Foucault 2002: 176). Die Geschichte ist demnach nichts anderes als die Aufeinanderfolge von herrschenden Ordnungen. Der Wechsel von Ordnungen besteht in der Bemächtigung von Regelapparaten durch Deutungen und Umdeutungen. Foucault fasst hier also Wissensordnungen als Herrschaftssysteme auf, wobei er die Vorstellung von »reineren« oder »besseren « Zuständen verwirft (Granjon 2005: 23). Das ist strukturidentisch mit dem Weberschen Gedanken der legitimen Ordnungen, die historisch aufeinanderfolgen und deren Veränderungen das Resultat von sozialen Kämpfen sind. Was in der späteren Sozialtheorie Diskurs genannt wird, heißt eben bei Weber noch »Kampf zwischen Werten«, der allerdings »mit Schallwellen und Tintentropfen« ausgetragen wird (Weber 1988: 354). Ähnlich implizit ist die Konvergenz der Grundauffassungen von Macht und Herrschaft wohl bei Bourdieu zu lesen.

\subsection{Pierre Bourdieu - symbolische Verkleidungen}

Auch Pierre Bourdieus Machtbegriff ist nicht einfach zu fassen, weil das Wort in seinem Werk zunächst nicht zentral zu sein scheint. Tatsächlich aber teilt Bourdieu mit Weber ein Verständnis von politischer Ordnung, das dem von Marx sehr nahe ist. Macht erscheint bei Bourdieu nämlich in verschiedenen Formen von Kapitalien. Unter der hier verfolgten Frage mutet es wie eine Schwäche seiner Konzeption an, dass er das symbolische Kapital nicht gegenüber anderen hervorhebt. Denn im Grunde ist es diese Form von Kapital, die in der Bourdieuschen Theorie die Funktion der Legitimität hat: Die Verfügungsgewalt über die anderen Kapitalien, die historisch immer irgendwie kontingente Verteilung, wird mit symbolischem Kapital umkleidet. Das meint die Legitimität einer Ordnung. Für Bourdieu wird durch die Generierung symbolischen Kapitals aus Macht Herrschaft. Mit Marx und Weber teilt er, dass die symbolische Ordnung des Staates, vor allem in Gestalt des Rechts, dieses Verhältnis betrifft. In dem knappen Aufsatz »De la maison du roi à la raison d'État« beschreibt Bourdieu (1997) die Genese dieser Form von symbolischem Kapital des modernen Staates, mit der nicht zuletzt auch die Juristen die Privilegien aller Amtsinhaber, sie selbst eingeschlossen, schaffen und zugleich mit sprachlicher Symbolik absichern. 
Mit symbolischem Kapital umkleidet, wird die Kontingenz der Kapitalverteilung unsichtbar, sie wird Herrschaft. Herrschaft ist auch bei Bourdieu das Selbstverständliche, schon nicht mehr Thematisierte im Habitus der Beteiligten. Die berühmte Formulierung Bourdieus (1998: 93), dass »wir von einem Staat gedacht werden, den wir zu denken meinen «, bezieht sich genau auf diesen Zusammenhang.

Die Konvergenz der Bourdieuschen Politikkonzeption mit der hier behaupteten Grundunterscheidung wird noch deutlicher, wenn man seine Arbeiten zum politischen Feld genauer in den Blick nimmt. Auch bei Bourdieu stehen nicht Entscheidungen im Zentrum des analytischen Interesses, sondern seine Soziologie der Politik interessiert sich vor allem dafür, wie Verteilungen und Hierarchisierungen legitimiert werden. Idées forces (Bourdieu 2001: 51), als Leitideen, sind es, mit denen legitime Ordnungen bewahrt werden, die ihrerseits Kapitalverteilungen begründen. Im politischen Feld ist das enjeux, also der Einsatz, um den des geht, die Legitimierung von Weltsichten, die mit Worten begründet, aber auch bestritten werden. In der Politik geht es um Macht, und die Form der Auseinandersetzung ist ein Streit um Definitionen und Klassifikationen, um Weltsichten (Gilcher-Holthey 2005: 189). Der Konflikt, der Streit um Legitimitäten, steht damit auch bei Bourdieu im Zentrum der Politik.

Zum Abschluss dieses Abschnitts seien die Kernpunkte der hier als Konvergenz von mehreren »klassischen« Autoren der politischen Soziologie noch einmal zusammengefasst:

Die Analyse politischer Phänomene kann sich nicht, wie im Governance-Paradigma, auf die Erfassung von Regelsetzungsmechanismen und der Zusammenhänge in und zwischen Institutionen beschränken. Das wird der global und historisch beobachtbaren Vielfältigkeit von Politik nicht gerecht und führt zu theoretischen Verengungen, großen blinden Flecken. Natürlich sind Institutionen sowie die in und um sie handelnden Akteure ein zentraler Gegenstand der Sozialwissenschaften. Doch statt die Analyse und Theoretisierung an die Erwartungserwartung der Governance als Regulierung auszurichten, wurde hier der Konflikt als analytischer Ansatzpunkt einer alternativen Perspektive vorgeschlagen.

Mit dem Begriff des Konflikts wurde die Sphäre des Politischen gekennzeichnet, in der divergierende und sich widersprechende Ansprüche auf Legitimität kommuniziert werden. Der Streit der Legitimitäten, der in Rede und Handeln stattfindet, wurde dabei als Zwischenraum zwischen bloßer Macht und ihrer legitimen Form, der Herrschaft, identifiziert. Dabei wird nicht von einem universellen Akteursmodell ausgegangen, sondern von sich wandelnden Subjektformen, deren Veränderungen selbst Resultat und Ausdruck veränderter Herrschaftsformen sind. Deshalb muss eine Soziologie des Politischen historisch sein. 
Denn auch wenn die Rekonstruktion der Genese von Institutionen, Subjekttypen oder Figurationen nicht notwendig über aktuelle Zusammenhänge Auskunft gibt, so ist die historische Verortung und die Auseinandersetzung mit der Geschichtlichkeit sozialer Konflikte vor allem aus zwei Gründen unverzichtbar: Nur die Analyse der akkumulierten Geschichte legt den Zugang zu der Thematisierung des Selbstverständlichen, des schon nicht mehr Thematisierten. Und ohne historische Perspektive ist es nicht möglich, die subjektiven Welten zu rekonstruieren, in denen sich Erfahrungen einlagern, auch wenn diese kein kohärentes Abbild des Vergangenen sind.

Wie im Folgenden gezeigt werden soll, eröffnet die historische Soziologie des Politischen, die den Konflikt als analytischen Ausgangspunkt nimmt, auch neue Perspektiven in der sozialwissenschaftlichen Forschung. Diese Impulse erstrecken sich nicht nur auf die angestammten Gebiete der Friedens-und Konfliktforschung, sondern liegen oft quer zu den etablierten Fachgrenzen.

\section{Der neue Blick einer historischen Soziologie des Politischen}

In diesem Beitrag wurde argumentiert, dass im Unterschied zu Governance- oder anderen funktionalistischen Ansätzen in der wissenschaftlichen Auseinandersetzung mit Politik eine historische Soziologie, die vom Konflikt aus denkt, besondere Vorzüge hat. Bisher wurde diese Perspektive indes nur begrifflich entfaltet und zu einigen klassischen Autoren in Bezug gesetzt. Worin der analytische Gewinn dieser Perspektive liegen könnte, soll in diesem Abschnitt diskutiert werden.

Die Konvergenzbewegung in der politischen Soziologie auf die Dynamiken von Macht und Herrschaft als zentrale begriffliche Unterscheidung nimmt den sozialen und politischen Konflikt zum analytischen Ausgangspunkt. Damit eröffnet sie auch die Chance auf eine interdisziplinäre Analytik der Macht. Die Beiträge hierzu kämen gewiss nicht nur aus der Politikwissenschaft, sondern auch aus der Geschichte und aus der Ethnologie ${ }^{16}$ - der political anthropology.

Die historische Soziologie des Politischen kann dabei an Forschungsfelder anknüpfen, in denen sich die Disziplinen schon lange begegnen. Um nur zwei herauszugreifen: Die Ansätze der Alltagsgeschichte und die mikropolitischen Beschreibungen der Ethnologie sind für ein verbessertes Verständnis von Macht, Herrschaft und Legitimität mutmaßlich besonders auskunftsreich. Der Alltag, le

16 Diese Interdisziplinarität geschieht auch als Import von Methoden; vgl. z.B. zur Ethnographie in der Politikwissenschaft die Beiträge in Schatz (2009) oder die Thematisierung von Politik in der political anthropology (Ferguson 1990) wie auch in der »Anthropologie des Staates« (Bierschenk 2008). 
politique par le bas, wie dies Jean-François Bayart, Achille Mbembe und Comi Toulabor nennen (2008), ist für die Erklärung von Politik ein bis heute vernachlässigtes Thema, obwohl wahrscheinlich alle politischen Soziologen zustimmen würden, dass der Staat, aber auch andere Formen von Herrschaft nur durch die Praktiken und Diskurse des Alltags reproduziert werden. Genau darin liegt das Banale und zugleich Mysteriöse der Herrschaft: Der Gehorsam ist das Erklärungsbedürftige, über seine Mechanismen wissen wir am wenigsten.

An einem zweiten Feld lässt sich zeigen, dass durch die Analyse lokaler Verhältnisse mehr Erkenntnis gewonnen werden kann als durch eine auf Steuerungsmöglichkeiten konzentrierte Perspektive: Das Zusammenspiel von legitimierenden Diskursen und disziplinierender Macht wird am Alltag der Sicherheitspolitik vielleicht am anschaulichsten sichtbar: Daran wird erkennbar, dass staatlichen Instanzen der sozialen Kontrolle zwar historisch eine zunehmend wichtige Rolle zukommt (vgl. Lüdtke 1992), das aber die entstandenen Herrschaftsbeziehungen keineswegs auf das Handeln staatlicher Agenturen reduziert werden können. Die konkreten Verhältnisse sind immer das Resultat der Interaktionen zwischen Regierungen, teilautonomen Agenturen und den »Objekten« ihrer Politik, wie Michel Fassin (2011) mit einer eindrucksvollen Ethnologie der französischen Polizei, ihren Legitimationsnöten und -konflikten in den Pariser banlieues gezeigt hat.

Auch eine noch so feinkörnige Analyse der Herrschaft wird immer die Schwierigkeit haben, das Nichtthematisierte überhaupt erst einmal zu erkennen und in seiner Bedeutung für die Geltung einer Ordnung zu erfassen (Foucault 1987: 245). An den genannten Arbeiten zeigt sich aber, warum der Konflikt so ein beliebtes Thema der politischen Soziologie ist. In Konflikten wird Vieles sichtbar, weil sie soviel zeigen: wie Herrschaft erodiert, wie mit Macht herausgefordert wird, und wie die neue Macht sich legitimiert und ihrerseits zur Herrschaft gerinnt. Das klingt nach vertrauten Themen, doch wie genau die Mechanismen der Legitimierung und Delegitimierung funktionieren, darüber geben die politikwissenschaftlichen Lehrbücher keine hinreichende Auskunft.

Der mögliche Gewinn der hier vorgeschlagenen Perspektive soll nachfolgend an drei Themen erläutert werden. Zunächst erlaubt sie ein besseres Verständnis der Prozesse der Internationalisierung von Herrschaft. Denn die Vertreter einer GlobalGovernance-Perspektive beziehen sich ja durchaus auf real beobachtbare Entwicklungen. In ihrem Paradigma sind nur die entscheidenden Merkmale dieses Prozesses nicht formulierbar.

Das zweite Thema betrifft die analytische Perspektive, mit der gerade dieser Prozess der Internationalisierung untersucht werden kann. Der Vorschlag besteht darin, das Globale nicht im abstrakt-allgemeinen Konzeptionellen zu thematisieren und 
die Empirie nicht auf Aggregatdaten zu reduzieren, sondern vom Lokalen auszugehen, die Einzelheit als Besonderung des Allgemeinen aufzufassen, und an ihr die konkrete Realität der internationalisierten Prozesse der Institutionalisierung von Macht und Deinstitutionalisierung von Herrschaft zu studieren. Erst aus dem theoretisch informierten Vergleich solcher Studien des Konkreten sind dann Verallgemeinerungen möglich. Aus einer wie auch immer gearteten reinen Theorie sind sie nicht deduzierbar.

Das dritte Thema schließlich ist die Frage des Gehorsams. Denn aus einer Perspektive auf Politik, die vom Konflikt als dem Regelfall aus denkt, gerät das bisher Selbstverständliche als Rätsel in den Blick. Warum ist nicht überall Konflikt? Warum werden Regeln befolgt, selbst solche, die aus einer Beobachterposition den Interesselagen der Folgsamen widersprechen?

\subsection{Die Internationalisierung von Macht und Herrschaft}

Aus der Perspektive einer historischen Soziologie des Politischen lassen sich die weitgehend ahistorisch geführten Diskussionen der Internationalen Beziehungen (IB) noch einmal auf fruchtbare Weise reformulieren. ${ }^{17}$ Denn wenn die internationale Geschichte auch nicht eine Geschichte der Klassenkämpfe ist, so ist sie doch eine Geschichte von Konflikten und damit eine Geschichte von Macht, Herrschaft und Legitimität. So wie sich, mit Norbert Elias gesprochen, die Figurationen ändern, in denen Menschen leben, so ändern sich auch die Legitimitäten, die geglaubten Geltungsgründe politischer Ordnung, die dennoch stets umstritten bleibt (vgl. Elias 1985).

Die Geschichte dieser Konflikte ist der gemeinsame Gegenstand der Sozialwissenschaften, den sie mit unterschiedlichen Methoden und Schwerpunkten erforschen. Nach dem Ende teleologischer Geschichtsauffassungen ist es unmodern geworden, Aussagen über große Prozesse zu treffen. Doch aus der hier vorgeschlagenen Perspektive einer historischen Soziologie des Politischen würde vielleicht erkennbar, dass diese Geschichte des Politischen kein endloses Nullsummenspiel ist, sondern eine Geschichte der Intensivierung und Akkumulation und Verdichtung der Macht, in deren Verlauf sich auch die Subjekte verändern, die an diesen Projekten teilhaben und ihnen zugleich unterworfen sind.

17 Eine solche veranschaulichende Übertreibung ist notwendig ungerecht. Natürlich gibt es auch in »den IB « historisch informierte Ansätze. Sie sind aber weder in Deutschland noch andernorts dominant. Zu denken wäre etwa an hegemoniekritische Ansätze, an Forschungen, die von Michel Foucault inspiriert sind, oder an die genderbezogene Forschung. Überblicke hierzu in Schieder/ Spindler (2010). 
Der säkulare Prozess der Globalisierung staatlicher Herrschaft deutet darauf hin. Er ist der bis heute unabgeschlossene konfliktive Prozess, weil sich vor- und nichtstaatliche Legitimitätsformen erhalten haben (vgl. Migdal 1988; Schlichte 2005) und zugleich neue, den Staat übergreifende Herrschaftsinstanzen entstehen. Die Ansprüche des Staates und seine Ausgestaltung stehen deshalb global im Zentrum politischer Konflikte.

Vielleicht ist aber die Entgegensetzung von Staat und Gesellschaft oder von Staat und »Globalisierung« trügerisch, weil sich nicht bloß Gewichtungen verschieben. Die lange Geschichte der Herrschaft, besonders aber seit dem strukturbildenden 19. Jahrhundert (vgl. Bayart 2004; Osterhammel 2009; Schlichte 2010) zeigt, dass sich auch jenseits des Staates die Machtbeziehungen verdichten und dass sich politische Herrschaft globalisiert. Das spricht dafür, dass diese Internationalisierung von Herrschaftsbeziehungen kein Nullsummenspiel ist, sondern eine tatsächliche Zunahme der Kontroll- und Sanktionsformen, die auch neue Subjektivitäten hervorbringen.

Damit wäre ein Gegenstandpunkt zum moralisch-technologischen GlobalGovernance-Paradigma gewonnen, der statt der Frage nach den Möglichkeiten weiterer internationaler Steuerungsmöglichkeiten die nach den Legitimitätsformen der Zunahme von Herrschafts- und Kontrollinstanzen und ihrer moralischen Produktion stellt. Immer größer, so scheint es, wird die Zahl der Institutionen, die an der Regelproduktion beteiligt sind. Das Band reicht von den Standards der internationalen Organisationen, über die EU als »supranationales Gebilde« oder das humanitäre Monitoring durch Nichtregierungsorganisationen bis hin zu Unternehmensethiken, staatlichem Recht und moralischer Kontrolle durch Amts- und Pfingstkirchen. Die moralischen Bezüge der Herrschaft werden immer internationaler, und über das Zusammenspiel der Institutionen und Zwangsapparate wird, so scheint es, die Kontrolle immer enger.

Aus einer herrschafts- und konfliktsoziologischen Perspektive entstehen neue analytische Fragen: Wie funktioniert in internationalen Herrschaftsfigurationen Gefolgschaft und Fügsamkeit? Entwickelt sich, analog zur »Autonomie des Staates « (vgl. Mann 1984; Poulantzas 1978), auch hier eine Verselbständigung formal-rationalisierter, meist bürokratischer Herrschaft gegenüber identifizierbaren Interessengruppen? Lässt sich dies in internationalen und supranationalen Organisationen beobachten? Auf welchen Legitimitäten beruht diese Herrschaft? Gibt es nicht doch »Trägerschichten« dieser Prozesse? Sind sie innerhalb der Apparate oder außerhalb derselben zu suchen?

Die Internationalisierung der Herrschaft betrifft aber auch Bereiche und Organisationen, die bisher nicht Gegenstand der Untersuchungen waren. Es gibt keine Politik ohne Macht. Die Ausweitung des Machtbegriffs, die Anerkennung ihrer 
Ubiquität - bei Weber wie bei Foucault oder Elias und Bourdieu - eröffnet auch neue Perspektiven für die Politikwissenschaft, die das Politische des vermeintlich »Privaten « nun in den Blick nehmen kann. Bislang sind beide nach Ebenen getrennt untersucht worden. Der Anteil von Berufsgruppen wie Anwälten, Ärzten, Sozialarbeitern und Lehrern an der Regierung der Welt wird aus dieser Perspektive ebenso thematisierbar wie die Rolle von Verwandtschaftssystemen, Freundschaften und anderen Instanzen sozialer Kontrolle (vgl. Brass 2000: 319).

Der Streit der Legitimitäten dauert zugleich an, weil die internationalisierte Herrschaft nicht konkurrenzlos umstritten bleibt. Auf nationaler Ebene wird zwischen Parteien und Verbänden um Verteilungen und Positionen, um Interessen und Werte, um Geltungsgründe überhaupt gestritten. Vor allem außerhalb der OECD-Welt geraten materiale Rationalitäten unter den Druck formaler Rationalisierung, der mit ausgreifenden bürgerlich-kapitalistischen Vergesellschaftungsformen einhergeht (vgl. Siegelberg 1994; Jung et al. 2003). Zwischen Staaten herrscht Uneinigkeit, welche politischen Gebilde und Akteure als Staaten anerkannt, politisch und juristisch legitimiert werden können (vgl. Isachenko 2012; Schlichte 2009: Kap. 4). Und in supranationalen Formen, internationalen Organisationen und auch in der Kooperation demokratischen Staaten bleibt das Problem, dass der demokratische Input über so viele Stationen vermittelt ist, dass der Gegensatz zwischen konstituierender Macht und konstitutioneller Form (vgl. Tully 2007) allzu offensichtlich wird. Der Unterschied zur Kongressdiplomatie des 19. Jahrhunderts ist nicht mehr erkennbar.

Dabei darf die Frage nicht vorschnell beantwortet werden, welcher Ordnung Legitimität zuzusprechen ist. Im Unterschied zum juristischen Legitimititätsbegriff ist dies auch im internationalen Feld keine normativ-deduktive Entscheidung, sondern eine empirische Frage (vgl. Biegoń et al. 2010). Die wissenschaftliche Aufgabe besteht auch hier darin, die Genese und Geltung dieser Legitimitäten am Material zu zeigen. Sind internationale Organisationen wirklich legitim? Wie lassen sich im Feld internationaler Politik Zwang, Eigeninteresse und Legitimitätsglaube als Gründer der Regelbefolgung empirisch unterscheiden (vgl. Hurd 1999)? Die jüngst begonnene geschichtswissenschaftliche Beschäftigung mit internationalen Entwicklungsorganisationen erweckt jedenfalls Zweifel an einer allzu idealistischen Interpretation (vgl. Unger 2010).

Schließlich lassen sich aus der hier umrissenen Perspektive auch zwischenstaatliche Konflikte soziologisch informierter adressieren. Denn Staaten stehen sich nicht einfach als rationale Akteure gegenüber, sondern als Aggregate von Agenturen, die alle eine historische Formation durchlaufen haben. In ihren Haltungen und Handlungen drücken sich die in ihrem Innern geronnenen Formen und Vorstellungen aus, die der soziologischen Analyse bedürfen und nicht einfach aus theoreti- 
schen Modellen abgeleitet werden können. Es sind diese subjektiv legitim erscheinenden Weltsichten, die in zwischenstaatlichen Konflikten, auch innerhalb von internationalen Organisationen, aufeinandertreffen. Der einfache Verweis auf »die Geschichte« reicht aber zur Erklärung nicht aus, wenn die Wissenschaft zum wirklichen Verständnis dieser Konflikte beitragen will. Doch wie viel ist über die Formierung des historischen Habitus der außenpolitischen Akteure und über den historischen Wandel dieses Feldes bekannt?

\subsection{Eine neue Analytik des Globalen über das Lokale}

Jeder wirkliche Konflikt ist konkret und am empirischen Primärmaterial als Gegenwiderspruch nachweisbar. Es ist sicher kein Zufall, dass alle im ersten Teil dieses Beitrags genannten Leittheoretiker zugleich intensiv an empirischen Gegenständen gearbeitet haben, um zu theoretischen und begrifflichen Schöpfungen zu gelangen, die über ihren Entstehungskontext hinaus für die empirische Forschung fruchtbar geblieben sind. Marx' frühe journalistische Tätigkeit und seine historischen Analysen, Webers Enqueten, die intensiven historischen Studien Foucaults und Elias' sowie die aufwendigen soziologische Erhebungen von Elias und Bourdieu sind für ihre theoretischen Beiträge konstitutiv.

Fortschritte in einer Analytik der Macht können offenbar nicht durch eine bloß theoretische Arbeit erzielt werden. Nur durch die Arbeit am Gegenstand, durch die Auseinandersetzung mit dem Material, d.h. die Analyse von empirisch vorfindlichen Phänomenen der Macht (vgl. Foucault 1987: 243-244), kann dieser Fortschritt erzielt werden. Die Dialektik von Begriff und Gegenstand gilt eben auch für den Begriff der Macht. Daraus scheint sich die Schwierigkeit zu ergeben, dass unter den Bedingungen der Globalisierung eine Analytik der Macht nur noch internationale, und damit auf den ersten Blick abstrakte Verhältnisse untersuchen müsste. Denn Machtbeziehungen und Herrschaftsverhältnisse lassen sich immer weniger unilokal konzeptionalisieren. Eine plurilokale Perspektive muss aber nicht notwendigerweise abstrakt sein. Globale Machtbeziehungen lassen sich nämlich durchaus auch an lokalen Verhältnissen aufzeigen. Auf diese Herausforderung hat nicht die politikwissenschaftliche, aber die soziologische und ethnologische Forschung schon reagiert.

Aus soziologischer Perspektive hat z.B. Alexander Veit (2010) am Fall der UNIntervention in Ituri die Verschränkung von globalen Machtbeziehungen und lokalen Konflikten veranschaulicht. Auch die Ethnologie hat mittlerweile plurilokale Machtdynamiken im Blick, wie sie etwa am Beispiel der Nuer im Süden des Sudans und in Des Moines, Illinois, aufgezeigt hat (Falge 2011). Denn das Lokale muss 
nicht, wie in der Globalisierungsliteratur üblich, als das Gegenteil des Globalen aufgefasst werden. Man kann es ebenso, ganz dialektisch, als die Besonderung des Allgemeinen begreifen. Die Alternative zum vielgeschmähten methodologischen Nationalismus ist nicht die Flucht in das abstrakte Globale, sondern das genaue Studium der lokalen Verhältnisse und ihrer Veränderungen. Den einzelnen Ort kann man eben auch als Produkt externer Beziehungen denken (Berking 2006), als Resultat von internationalen Machteffekten und Herrschaftsstrukturen verstehen.

Das Versprechen dieser analytischen Perspektive, die vom Lokalen ausgeht, wäre es auch, diejenigen Sinngehalte, Wertbezüge und Praktiken aufzudecken, die der deduktiven Variablenlogik des sozialwissenschaftlichen Mainstreams nicht in den Blick geraten können, weil sie an Problematisierungen orientiert ist, die in westlichen Kontexten, vor allem die USA, als Eigenbeobachtungen generiert wurden (Chabal/Daloz 2006: 5). Eine Semiotik des Lokalen müsste zuerst versuchen zu ermitteln, in welchen Begrifflichkeiten die Akteure selbst ihre Wirklichkeit erfahren und bewerten, anstatt vorgefertigte Schablonen der Strukturierung für die Analyse zu verwenden, die keine Sensibilität gegenüber der subjektiven Seite der sozialen Welt mehr besitzen. Denn es sind diese lokalen Kategorien und Schemata, die darüber entscheiden, welche der von außen wahrgenommenen Widersprüche letztlich handlungsleitend und damit konfliktgenerierend wirken.

\subsection{Der unselbstverständliche Gehorsam}

Aus dieser hier vorgeschlagenen Perspektive wird die Normalität politischer Herrschaft noch einmal anders befragt. Das gilt in den Sozialwissenschaften heute vor allem für die aktuellen Formen staatlicher Herrschaft in westlichen Staaten, in denen Fügsamkeit selbstverständlich geworden zu sein scheint und vielleicht eben deshalb in vielerlei Hinsicht gar nicht untersucht ist. Zwar ist auch für moderne Staaten davon auszugehen, dass sich in ihnen Anteile der von Weber herausgearbeiteten idealtypischen Legitimität finden. Jüngere phänomenologische Studien zeigen jedoch immer wieder, wie überraschend unbekannt diese Herrschaftsbeziehungen sind (vgl. Abélès 1990; Paris 2005). Erkennbar werden sie oft erst im Konflikt.

$\mathrm{Zu}$ untersuchen, worauf Gefolgschaft und Gehorsam beruhen, ist wegen einer zunehmenden Reduktion auf eine utilitaristische Anthropologie in weiten Teilen der Sozialwissenschaften ebenso aus dem Blickfeld geraten, wie die genaue Bestimmung der Legitimitätsdynamiken im Konflikt. Vielleicht liegt es an dem übergroßen Erbe strukturfunktionalistischen Denkens in der Politikwissenschaft, dass Integration und Stabilität als nicht fragwürdiger Normalfall gelten. Aus einer herrschaftssoziologischen Perspektive, die konfliktorientiert ist, sieht das hingegen anders aus. 
Die Kreativität der Menschen, wechselnde Einflüsse aus sich selbst wandelnden Umgebungen und Veränderungen der Akteure selbst lassen hier vielmehr den Konflikt als Normalfall erscheinen. Das problemlose Regieren wird dagegen zum Rätsel: Wie ist die massenhafte Gefolgschaft und die Regelbefolgung eigentlich zu erklären? Die »Macht des Gehorsams « (Hibou 2006) gerade in autoritären und totalitären Zusammenhängen wird zu einer eher beunruhigenden Größe. Die Herstellung von Gehorsam und Fügsamkeit lässt sich nur teilweise als Arbeit der Disziplinierung begreifen. Verständlich wird sie erst, wenn die Arbeit der Legitimierung für diejenigen politischen Verhältnisse mit in den Blick genommen wird, die gemeinhin als bar jeder Legitimität angesehen werden (vgl. Hibou 2011). Die Theorie der Herrschaft als legitime Macht hat damit den weiteren Vorzug, ein weithin übersehenes Thema wieder in den Blick zu rücken, nämlich die Herstellung von Fügsamkeit, von Gehorsam. ${ }^{18}$

Auch jenseits autoritärer Herrschaft hat diese Frage ihre Aktualität. Der soziale Konflikt ist schließlich kein Thema, das nur die südlichen Weltregionen oder vergangene Epochen betrifft. In weiten Teilen der OECD ist die Repolitisierung nach Umbau des Staates aber offensichtlich ausgeblieben (Schäfer 2008: 38). Aus der Perspektive einer historischen Soziologie des Politischen lässt sich hier die Diskussion um die Postdemokratie (Crouch 2004; Crenson/Ginsberg 2002) mit der These von der »Verwilderung der sozialen Konflikte« (Honneth 2011: 45) in Verbindung bringen. Welche legitimatorischen Folgen haben die Veränderungen der institutionellen Landschaft westlicher Staaten? Inwiefern hat die Umstellung auf Expertenherrschaft in Netzwerken, ganz im Sinne der Governance, einen Effekt auf die Anund Abwesenheit von sozialen Konflikten? Oder verbirgt sich hinter dem Eindruck der Fügsamkeit und der Entpolitisierung nur eine schleichende Verschiebung der Konfliktlinien?

\section{Die ewig offenen Fragen der Friedens- und Konfliktforschung}

Jedes Fach und jede Forschungsrichtung wird über ihre Kernfragen konstituiert. Auch wenn die Friedens- und Konfliktforschung bis heute keinen Fachcharakter angenommen hat, so sind doch auch für sie zwei Fragen konstitutiv: die Frage nach dem theoretischen Ort der Gewalt und die Frage nach der Normativität der je eigenen

18 Sieht man von kritischen älteren Beiträgen ab, wie Étienne de la Boéties »De la servitude volontaire« (1574) und Friedrich Nietzsches »Genealogie der Moral« (1887) ist das Thema der Fügsamkeit oder des Gehorsams eher in sozialtechnologische Compliance-Forschung gemündet, die keinen kritischen Impuls mehr hat, sondern die »Effizienz«-Gesichtspunkte der von ihr untersuchten Großinstitutionen in der Regel ohne Aufforderung übernimmt. 
Forschung. Über beide Fragen kann nicht abschließend entschieden werden, aber sie erzwingen eine Positionierung jedes Beitrags, der Legitimität erheischen möchte. Was wären die Antworten auf diese Fragen aus der Perspektive einer historischen Soziologie des Politischen?

Die historische Soziologie des Politischen müsste zunächst der Gewalt den Ort in der Theorie der Politik gewähren, der ihr bisher verwehrt wurde. Sieht man von normativen Theorien ab, die das Gewaltproblem für die Begründung von Ordnung anführen, so ist der empirische Zusammenhang von Gewalt und Legitimität jedoch noch ein Randthema der Theorie von Politik und Gesellschaft geblieben. ${ }^{19}$ Die historische Soziologie des Politischen könnte helfen, diese fast skandalös anmutende Abwesenheit der Gewalt in der jüngeren Sozialtheorie (Joas/Knöbl 2008) zu überwinden, ohne physische Gewalt und Zwang als Reste vormoderner Politik zu interpretieren. Dafür sind bereits jetzt die Erkenntnisse über die Gewaltgeladenheit von Modernisierungsprozessen (vgl. Jung et al. 2003) und über das Kriegsverhalten westlicher Demokratien (vgl. Geis et al. 2007) zu auffällig.

Auch die Figuren der Gewalt im Innern von modernen westlichen Gesellschaften müssten aus dieser Perspektive noch einmal in den Blick genommen werden. Tyrell (1980) hat die zentrale Rolle des Zwangs als Herrschaftsmittel hervorgehoben, die alle Formen legitimer Macht begleitet. Noch die stabilsten politischen Systeme wenden Zwangsmittel an, wenn dies von Apparaten und politischen Repräsentanten für erforderlich gehalten wird. Während die Gleichung zwar im Groben zu stimmen scheint, dass sich Zwangsgewalt und Legitimität politischer Herrschaft in einem Nullsummenverhältnis zueinander verhalten, weil der Gebrauch physischer Gewalt immer - und seien es noch so begrenzte - delegitimierende Effekte hat, so sind zwangsfreie Herrschaftsgebilde seltene historische Ausnahmen wie rein gewaltbasierte politische Macht, wenn es sie überhaupt gegeben hat. Zwei Fragen resultieren aus dieser Perspektive zum theoretischen Ort der Gewalt: Unter welchen Bedingungen und in welchen Konstellationen wird der Einsatz physischer Gewalt zum Legitimitätsproblem, und zwar sowohl für die Akteure wie für ihre Beobachter? Und zweitens: Welche Äquivalente lassen den Einsatz von physischer Gewalt dysfunktional erscheinen und ersetzen sie als Mittel sozialer Kontrolle?

Die zweite offene Frage, die aus der kritischen Tradition der Friedens- und Konfliktforschung an die historische Soziologie des Politischen zu richten wäre, betrifft ihre Normativität. Denn die historische Soziologie des Politischen, die hier als Konvergenz behauptet wurde, wirkt normativ unbefriedigend. Wie hoch diese Enttäu-

19 Zu diesem grundlegenden Zusammenhang vgl. Collins (1986: 155; 2007), Breuer (2007) und Schlichte (2009). 
schung ausfällt, das hängt allerdings von der Höhe der Erwartungen ab. Normative Leitsterne und Leitbegriffe kann sie nicht bieten. Aus der Sicht Webers und Foucaults sind solche Leitsterne, wie sie Hannah Arendt und Jürgen Habermas formulieren, natürlich durch und durch bürgerliche Politikauffassungen, an die sie seit ihrer Lektüre von Marx und Nietzsche nicht mehr glauben können (Colliot-Thélène 2001: 280). ${ }^{20}$ Es wird aus der Weberschen Herrschaftssoziologie aber erkennbar, woraus sich die emphatischen Machtbegriffe im Sinne der power to nähren, nämlich aus dem Typus der »nicht legitimen Herrschaft «, der mit der Geschichte der abendländischen Stadt assoziiert wird (vgl. Weber 1985: 727-814; Fitzi 2004: 176-182), in der sich der Bruch mit feudalen Banden als erstes vollzieht und in der sich eine sich selbst Gesetze gebende Bürgerschaft zusammenschließt, die mit unterschiedlichem Erfolg Unterwerfungsversuchen trotzt und zum Hort der Rationalisierung und Verrechtlichung politischer Herrschaft wird (vgl. Oexle 2001). In einem ganz historischen Sinne steht der emphatische Machtbegriff also tatsächlich in einer (stadt-)bürgerlichen Tradition. Ob seine normativen Ansprüche damit über seinen Entstehungskontext hinausreichen, ist indes eine offene Frage.

Deshalb muss die vorgestellte Perspektive, die als Konvergenz einer Reihe von Autoren plausibilisiert werden sollte, aber nicht normativ irrelevant sein. Ihre Normativität, oder besser ihr kritischer Gehalt, läge aber nicht in der Formulierung und Begründung einer bestimmten Norm, sondern in ihrer zunächst selbstkritischen und dann als immanente Kritik nach außen gerichteten Haltung (vgl. Schlichte 2006).

Die Rücksichtslosigkeit auch gegenüber den eigenen Illusionen, die Weber einfordert, die »Jagd auf Mythen«, die Norbert Elias zum Zweck der Soziologie gemacht hat, und die Offenlegung verdeckter Herrschaftsbeziehungen und der Mechanismen der Macht, wie sie bei Bourdieu und Foucault programmatisch werden, sind normativ ebenso effektvoll. Die Kritik und die Veränderung des Bestehenden wird in dieser Perspektive nur möglich, wenn das Bestehende offen analysiert wird. Dazu würde die Abschätzung der Machteffekte des eigenen Handelns ebenso gehören, wie die selbstkritische Reflexion darüber, an welchem Herrschaftsprojekt

20 Trotz der vordergründig ganz unterschiedlichen Begriffsbestimmungen, gerade beim Begriff der Macht, steht das Werk Arendts der hier vorgestellten Perspektive aber nicht unvermittelbar gegenüber. Das gilt vor allem für »Elemente und Ursprünge totalitärer Herrschaft« (Arendt 1962). Darin ist von Machtakkumulationen die Rede, wie sie etwa imperialistischen Expansionen zu eigen ist (Arendt 1962: 193-198). Dass damit die Verselbständigung von Machtgebilden gemeint ist, die Überblendung der tatsächlichen Verhältnisse, die ja Herrschaft sind, durch Truggebilde oder diffuse Vorstellungen, das wird in ihren Analysen bürokratischer und totaler Herrschaft unübersehbar. Überall dort, wo Arendt von der Bürokratie als »der Herrschaft des Niemand « (Arendt 2002: 57) spricht, scheinen ähnliche Unterscheidungen wirksam zu sein wie die hier vorgestellten. 
sich die eigene wissenschaftliche Tätigkeit beteiligt. Darin bestünde der erste und vielleicht wichtigste Schritt der Kritik.

\section{Literatur}

Abélès, Marc 1990: Anthropologie de l'Etat, Paris.

Anter, Andreas 2001: Von der politischen Gemeinschaft zum Anstaltsstaat. Das

Monopol der legitimen Gewaltsamkeit, in: Hanke, Edith/Mommsen, Wolfgang

J. (Hrsg.): Max Webers Herrschaftssoziologie, Tübingen, 121-138.

Arendt, Hannah 1962: Elemente und Ursprünge totaler Herrschaft, Frankfurt a.M. Arendt, Hannah 2002: Vita activa oder Vom tätigen Leben, München.

Barker, Rodney 1990: Political Legitimacy and the State, Oxford.

Bayart, Jean-François 2000: Africa in the World: a History of Extraversion, in:

African Affairs 99: 2, 217-267.

Bayart, Jean-François 2004: Le gouvernement du monde. Une critique politique de la globalisation, Paris.

Bayart, Jean-François/Mbembe, Achille/Toulabor, Comi 2008: Le politique par le bas en Afrique noire, 2. Auflage, Paris.

Benz, Arthur/Dose, Nicolai 2004: Governance - Modebegriff oder nützliches sozialwissenschaftliches Konzept?, in: dies. (Hrsg.): Governance. Regieren in komplexen Regelsystemen. Eine Einführung, 2. Auflage, Wiesbaden, 13-36.

Berking, Helmuth 2006: Raumtheoretische Paradoxien im Globalisierungsdiskurs, in: ders. (Hrsg.): Die Macht des Lokalen in einer Welt ohne Grenzen, Frankfurt a.M., 7-23.

Bhambra, Gurminder 2010: Historical Sociology, International Relations, and Connected Histories, in: Cambridge Review of International Affairs 23: 1, 127-143. Biegoń, Dominika/Gronau, Jennifer/Nonhoff, Martin/Nullmeier, Frank/Schmidtke, Henning/Schneider, Steffen 2010: Prekäre Legitimitäten. Rechtfertigung von Herrschaft in der postnationalen Konstellation, Frankfurt a.M.

Bierschenk, Thomas 2008: The Every-Day Functioning of an African Public Service. Informalization, Privatization and Corruption in Benin's Legal System, in: Journal of Legal Pluralism and Unofficial Law 57/2008, 101-139.

Börzel, Tanja 2010: Governance with/out Government. False Promises or Flawed Premises? (SFB-Governance Working Paper Series, No. 23, Berlin: SFB 700), in: http://www.sfb-governance.de/publikationen/sfbgov_wp/wp23/WP23_hp. pdf?1277900593; 16.11.2011.

Bogner, Artur 1989: Zivilisation und Rationalisierung. Die Zivilisationstheorien M. Webers, N. Elias' und der Frankfurter Schule, Opladen. 
Bonacker, Thorsten 2002: Sozialwissenschaftliche Konflikttheorien. Eine Einführung, Opladen.

Bonacker, Thorsten 2009: Konflikttheorien, in: Kneer, Georg/Schroer, Markus (Hrsg.): Handbuch soziologische Theorien, Wiesbaden, 179-198.

Bourdieu, Pierre 1997: De la maison du roi à la raison d'Etat, in: Actes de la recherche en sciences sociales 118: 1, 55-68.

Bourdieu, Pierre 1998: Praktische Vernunft. Zur Theorie des Handelns, Frankfurt a.M.

Bourdieu, Pierre 2001: Langage et pouvoir symbolique, Paris.

Brass, Paul R. 2000: Foucault Steals Political Science, in: Annual Review of Political Science 3/2000, 305-330.

Breuer, Stefan 1994: Bürokratie und Charisma. Zur politischen Soziologie Max Webers, Darmstadt.

Breuer, Stefan 2007: Wege zum Staat, in: Breuer, Stefan/Anter, Andreas (Hrsg.):

Max Webers Staatssoziologie. Positionen und Perspektiven, Baden-Baden, 57-80.

Chabal, Patrick/Daloz, Jean-Pascal 2006: Culture Troubles. Politics and the Interpretation of Meaning, Chicago, IL.

Clegg, Stewart R./Haugaard, Mark 2009: Introduction. Why Power is the Central Concept of Social Sciences, in: dies. (Hrsg.): The Sage Handbook of Power, London, 3-24.

Collins, Randall 1986: Weberian Sociological Theory, Cambridge, MA.

Collins, Randall 2007: Violence: A Microsociological Theory, Princeton, NJ.

Colliot-Thélène, Catherine 2001: Etudes wébériennes. Rationalités, histoires, droits, Paris.

Crenson, Matthew A./Ginsberg, Benjamin 2002: Downsizing Democracy. How America Sidelined it's Citizens and Privatized it's Public, Baltimore, MD.

Crouch, Colin 2004: Post-Democracy, London.

de la Boétie, Étienne 1574: Discours de la servitude volontaire, Paris 2002.

Dobner, Petra 2010: Wasserpolitik. Zur politischen Theorie, Praxis und Kritik globaler Governance, Frankfurt a.M.

Elias, Norbert 1970: Was ist Soziologie?, Weinheim.

Elias, Norbert 1985: Humana conditio. Beobachtungen zur Entwicklung der Menschheit am 40. Jahrestag eines Kriegsendes (8. Mai 1945), Frankfurt a.M. Elias, Norbert 1987: Die Gesellschaft der Individuen, herausgegeben von M. Schroeter, Frankfurt a.M.

Elias, Norbert 1990: Die höfische Gesellschaft. Untersuchungen zur Soziologie des Königtums und der höfischen Aristokratie, Frankfurt a.M. 
Elias, Norbert/Scotson, John L. 1990: Etablierte und Außenseiter, Frankfurt a. M. Falge, Christiane 2011: The Global Nuer: Transnational Lifelihoods, Religious Movements and War, Oxford.

Fassin, Didier 2011: La force de l'ordre. Une anthropologie de la police des quartiers, Paris.

Ferguson, James 1990: The Anti-Politics Machine: »Development«, Depoliticization, and Bureaucratic Power in Lesotho, Cambridge.

Fitzi, Gregor 2004: Max Webers politisches Denken, Konstanz.

Foucault, Michel 1987: Nachwort. Das Subjekt und die Macht, in: Dreyfus, Hubert

L./Rabinow, Paul (Hrsg.): Michel Foucault: Jenseits von Strukturalismus und Hermeneutik, Weinheim, 243-261.

Foucault, Michel 2002: Nietzsche, die Genealogie, die Historie, in: ders.: Dits et

Ecrits, Schriften in vier Bänden, Band 2, Frankfurt a.M., 167-191.

Foucault, Michel 2005: Analytik der Macht, Frankfurt a.M.

Fuchs, Dieter/Roller, Edeltraud 2007: Politik, in: dies. (Hrsg.): Lexikon Politik. Hundert Grundbegriffe, Stuttgart, 205-210.

Geis, Anna/Müller, Harald/Wagner, Wolfgang (Hrsg.) 2007: Schattenseiten des Demokratischen Friedens. Zur Kritik einer Theorie liberaler Außen- und Sicherheitspolitik, Frankfurt a.M.

Gilcher-Holtey, Ingrid 2005: Gegen Strukturalismus, Pansymbolismus und Pansemiologie. Pierre Bourdieu und die Geschichtswissenschaft, in: Colliot-Thélène, Catherine/François, Etienne/Gebauer, Gunter (Hrsg.): Pierre Bourdieu. Deutsch-französische Perspektiven, Frankfurt a.M., 179-194.

Gobin, Corinne 2007: Gouvernance, in: Durand, Pascal (Hrsg.): Les nouveaux mots du pouvoir. Abécédaire critique, Brüssel, 267-283.

Göhler, Gerhard 2009: >Power to< and >Power over`, in: Clegg, Stewart R./Haugaard, Mark (Hrsg.): The Sage Handbook of Power, London, 27-39.

Göhler, Gerhard 2011: Macht, in: Göhler, Gerhard/Iser, Matthias/Kerner, Ina (Hrsg.): Politische Theorie. 25 umkämpfte Begriffe zur Einführung, 2. Auflage, Wiesbaden, 224-240.

Granjon, Marie-Christine 2005: Penser avec Michel Foucault. Théorie critique et pratique politiques, Paris, 5-74.

Hagmann, Jonas 2011: Der Westen legt sich die Welt zurecht. Das Fach »Internationale Politik« erscheint als engstirniges Denksystem, in: Frankfurter Allgemeine Zeitung, 9. 11. 2011, 5.

Heinich, Nathalie 1997: La sociologie de Norbert Elias, Paris.

Hibou, Béatrice 2006: La force de l'obéissance. Economie politique de la répression en Tunisie, Paris. 
Hibou, Béatrice 2011: Anatomie politique de la domination, Paris.

Honneth, Axel 2011: Verwilderungen. Kampf um Anerkennung im frühen 20. Jahrhundert, in: Aus Politik und Zeitgeschichte 61: 1-2, 37-45.

Hurd, Ian 1999: Legitimacy and Authority in International Politics, in: International Organization 53: 2, 379-408.

Iggers, Georg G./Wang, Q. Edward/Mukherjee, Supriya 2008: A Global History of Modern Historiography, London.

Isachenko, Daria 2012: The Making of Informal States. Statebuilding in Northern Cyprus and Transdniestria, London.

Joas, Hans/Knöbl, Wolfgang 2008: Kriegsverdrängung. Ein Problem in der Geschichte der Sozialtheorie, Frankfurt a.M.

Jung, Dietrich/Schlichte, Klaus/Siegelberg, Jens 2003: Kriege in der Weltgesellschaft. Strukturgeschichtliche Erklärungen kriegerischer Gewalt, 1945-2000, Wiesbaden.

Kaven, Carsten 2006: Sozialer Wandel und Macht. Die theoretischen Ansätze von Max Weber, Norbert Elias und Michel Foucault im Vergleich, Marburg.

Kreide, Regina/Niederberger, Andreas 2011: Politik, in: Göhler, Gerhard/Iser, Matthias/Kerner, Ina (Hrsg.): Politische Theorie. 22 Umkämpfte Begriffe zur Einführung, Wiesbaden, 290-306.

Krippendorff, Ekkehart 1975: Internationales System als Geschichte. Einführung in die Internationalen Beziehungen, Frankfurt a.M.

Lehmbruch, Gerhard 1976: Parteienwettbewerb im Bundesstaat, Stuttgart.

Lemke, Thomas 2001: Max Weber, Norbert Elias und Michel Foucault über Macht und Subjektivierung, in: Berliner Journal für Soziologie 11: 1, 77-95.

Lüdtke, Alf (Hrsg.) 1992: »Sicherheit« und »Wohlfahrt«. Polizei, Gesellschaft und Herrschaft im 19. und 20. Jahrhundert, Frankfurt a.M.

Luhmann, Niklas 2010: Politische Soziologie, Frankfurt a.M.

Mann, Michael 1984: The Autonomous Power of the State: Its Origins, Mechanisms and Results, in: Achrives Européenes de Sociologie 25: 2, 185-213.

Marx, Karl 1953: Die Frühschriften, herausgegeben von Siegfried Landshut, Stuttgart.

Marx, Karl: Marx-Engels-Werke (MEW), Berlin.

Mayntz, Renate 2004: Governance im modernen Staat, in: Benz, Arthur (Hrsg.): Governance. Regieren in komplexen Regelsystemen, Wiesbaden, 65-76.

McFalls, Laurence 2008: Les fondements rationnels et sociaux des passions politiques. Vers une sociologie de la violence contemporaine avec Weber et Foucault, in: Anthropologie et Sociétés 32: 3, 155-172. 
McFalls, Laurence/Simard, Augustin/Thériault, Barbara 2007: Introduction: Towards a Conceptual Reception-History of Max Weber's Ouevre, in: McFalls, Laurence (Hrsg.): Max Weber's 〉Objectivity< Reconsidered, Toronto, 3-30.

Messmer, Heinz 2003: Der soziale Konflikt. Kommunikative Emergenz und systemische Reproduktion, Stuttgart.

Migdal, Joel S. 1988: Strong Societies and Weak States. State-Society Relations and State Capabilities in the Third World, Princeton, NJ.

Nietzsche, Friedrich 1887: Zur Genealogie der Moral. Eine Streitschrift, in: Kritische Studienausgabe, Band 5, herausgegeben von Giorgio Colli und Mazzino Montinari, München, 245-412.

Oexle, Otto Gerhard 2001: Priester, Krieger, Bürger. Formen der Herrschaft in Max Webers >Mittelalter८, in: Hanke, Edith/Mommsen, Wolfgang J. (Hrsg.): Max Webers Herrschaftssoziologie, Tübingen, 203-222.

Offe, Claus 2009: Governance - an »Empty Signifier«?, in: Constellations 16: 4, 550-562.

Osterhammel, Jürgen 2009: Die Verwandlung der Welt. Eine Geschichte des 19. Jahrhunderts, München.

Paris, Rainer 2005: Normale Macht. Soziologische Essays, Konstanz.

Pitkin, Hanna 1972: Wittgenstein and Justice, Berkeley, CA.

Popitz, Heinrich 1992: Phänomene der Macht, 2. Auflage, Tübingen.

Poulantzas, Nikos 1978: L'État le pouvoir, le socialisme, Paris.

Rancière, Jacques 2002: Das Unvernehmen. Politik und Polizei, Frankfurt a.M.

Reichelt, Helmut 2008: Das Verhältnis von Staat und Gesellschaft im Marxschen

Frühwerk, in: Hirsch, Joachim/Kannankulan, John/Wissel, Jens (Hrsg.): Der

Staat der bürgerlichen Gesellschaft. Zum Staatsverständnis von Karl Marx, Baden-Baden, 25-40.

Reynaud, Philippe 1996: Max Weber et les dilemmes de la raison moderne, Paris. Risse, Thomas 2007: Regieren in Räumen begrenzter Staatlichkeit. Zur »Reisefä-

higkeit« der Governance-Konzeptes (SFB-Governance Working Paper Series Nr. 5, FU Berlin), in: http://www.sfb-governance.de/publikationen/sfbgov_wp/ wp5/wp5.pdf; 10.01.2012.

Schäfer, Armin 2008: Krisentheorie der Demokratie. Unregierbarkeit, Spätkapitalismus und Postdemokratie (MPifG Discussion Paper 08/10), Köln, in: http:// www.mpifg.de/pu/mpifg_dp/dp08-10.pdf; 10.01.2012.

Schatz, Edward (Hrsg.) 2009: Political Ethnography. What Immersion Contributes to the Study of Power, Chicago, IL.

Schatzberg, Michael 1980: Politics and Class in Zaire: Bureaucracy, Business, and Beer in Lisala, New York, NY. 
Schieder, Siegfried/Spindler, Manuela (Hrsg.) 2010: Theorien der Internationalen Beziehungen, 3. Auflage, Opladen.

Schlichte, Klaus 2005: Der Staat in der Weltgesellschaft. Politische Herrschaft in Afrika, Asien und Lateinamerika, Frankfurt a.M.

Schlichte, Klaus 2006: Ist Kritik noch möglich? Zum Verhältnis von Politik und Wissenschaft in den Internationalen Beziehungen, in: Hellmann, Günther (Hrsg.): Forschung und Beratung in der Wissensgesellschaft. Das Feld der Internationalen Beziehungen und der Außenpolitik, Baden-Baden, 283-306.

Schlichte, Klaus 2009: In the Shadow of Violence: The Politics of Armed Groups, Frankfurt a.M.

Schlichte, Klaus 2010: Das formierende Säkulum. Macht und Recht in der internationalen Politik des 19. Jahrhunderts, in: Lappenküper, Ulrich/Markowitz, Rainer (Hrsg.): Macht und Recht. Das Völkerrecht in den internationalen Beziehungen, Paderborn, 161-177.

Schlotter, Peter/Wisotzki, Simone (Hrsg.) 2011: Friedens- und Konfliktforschung, Baden-Baden.

Senghaas, Dieter 1982: Von Europa lernen. Entwicklungsgeschichtliche Betrachtungen, Frankfurt a.M.

Siegelberg, Jens 1994: Krieg und Kapitalismus. Eine Theorie des Krieges in der Weltgesellschaft, Münster/Hamburg.

Tully, James 2007: Modern Constitutional Democracy and Imperialism, in: Loughlin, Martin/Walker, Neil (Hrsg.): The Paradox of Constitutionalism: Constituent Power and Constitutional Form, Oxford, 461-493.

Tyrell, Hartmann 1980: Gewalt, Zwang und die Institutionalisierung von Herrschaft. Versuch einer Neuinterpretation von Max Webers Herrschaftsbegriff, in: Pohlmann, Rosemarie (Hrsg.): Person und Institution. Helmut Schelsky gewidmet, Würzburg, 59-92.

Unger, Corinna 2010: Histories of Development and Modernization. Findings, Reflections, Future Research, in: http://hsozkult.geschichte.hu-berlin.de/forum/ 2010-12-001; 19.11.2011.

Veit, Alexander 2010: Intervention as Indirect Rule: Civil War and State Building in Ituri/DR Congo, Frankfurt a.M.

Weber, Max 1985: Wirtschaft und Gesellschaft. Grundriß der verstehenden Soziologie, 5. Auflage, Tübingen.

Weber, Max 1988: Gesammelte Politische Schriften, 5. Auflage, Tübingen.

Weber, Max 1991: Wirtschaftsgeschichte, 5. Auflage, Berlin.

Yildiz, Ersin 2008: Marx' verfassungstheoretische Schriften und die Entwicklung der materialistischen Rechtstheorie, in: Hirsch, Joachim/Kannankulam, John/ 
Wissel, Ines (Hrsg.): Der Staat der bürgerlichen Gesellschaft. Zum Staatsverständnis von Karl Marx, Baden-Baden, 116-134.

\section{Der Autor}

Prof. Dr. Klaus Schlichte, Institut für Interkulturelle und Internationale Studien, Universität Bremen

E-Mail: klaus.schlichte@iniis.uni-bremen.de 